\title{
Reexamining the Gradient and Countergradient Representation of the Local and Nonlocal Heat Fluxes in the Convective Boundary Layer
}

\author{
Bowen Zhou, Shiwei Sun, Kai YaO, AND Kefeng Zhu \\ Key Laboratory for Mesoscale Severe Weather, Ministry of Education, and School of Atmospheric Sciences, \\ Nanjing University, Nanjing, China
}

(Manuscript received 6 July 2017, in final form 20 March 2018)

\begin{abstract}
Turbulent mixing in the daytime convective boundary layer (CBL) is carried out by organized nonlocal updrafts and smaller local eddies. In the upper mixed layer of the CBL, heat fluxes associated with nonlocal updrafts are directed $u p$ the local potential temperature gradient. To reproduce such countergradient behavior in parameterizations, a class of planetary boundary layer schemes adopts a countergradient correction term in addition to the classic downgradient eddy-diffusion term. Such schemes are popular because of their simple formulation and effective performance. This study reexamines those schemes to investigate the physical representations of the gradient and countergradient (GCG) terms, and to rebut the often-implied association of the GCG terms with heat fluxes due to local and nonlocal (LNL) eddies. To do so, large-eddy simulations (LESs) of six idealized CBL cases are performed. The GCG fluxes are computed a priori with horizontally averaged LES data, while the LNL fluxes are diagnosed through conditional sampling and Fourier decomposition of the LES flow field. It is found that in the upper mixed layer, the gradient term predicts downward fluxes in the presence of positive mean potential temperature gradient but is compensated by the upward countergradient correction flux, which is larger than the total heat flux. However, neither downward local fluxes nor larger-than-total nonlocal fluxes are diagnosed from LES. The difference reflects reduced turbulence efficiency for GCG fluxes and, in terms of physics, conceptual deficiencies in the GCG representation of CBL heat fluxes.
\end{abstract}

\section{Introduction}

The daytime convective boundary layer $(\mathrm{CBL})$ is vigorously mixed via large and small turbulent eddies, as shown in Fig. 1 adapted from Hunt et al. (1988). Large columns of rising buoyant air, also known as updrafts or thermals, are primarily driven by surface heating. They can take on cellular or roll organizations, depending on the relative strength of the mechanical (i.e., vertical wind shear production) to thermal forcings [see Salesky et al. (2017) and references therein]. In the presence of strong surface heat flux and weak shear, coherent structures in the CBL are largely cellular, consisting of narrow spokes of strong updrafts and broad regions of compensating downdrafts (Schmidt and Schumann 1989). On the other hand, when wind shear is strong and surface heat flux is small, convection tends to organize into horizontal roll vortices with axes aligned within $10^{\circ}-20^{\circ}$ of the wind shear direction (Young 1988). The organized thermals transport momentum and heat across the depth of the CBL in an advective-like

\footnotetext{
Corresponding author: Kefeng Zhu, kefeng@nju.edu.cn
}

fashion and are thus referred to as nonlocal eddies (Stull 1988, chapter 11). In comparison, smaller local eddies are omnipresent in the CBL. In the surface layer (i.e., roughly the bottom $10 \%$ of the CBL), local eddies are generated mostly by wind shear, with sizes limited by their distance to the ground. In the stably stratified entrainment zone (i.e., top $15 \%-20 \%$ of the CBL), local eddies can form from breaking shear-instability waves (Stull 1988). In the mixed layer in between, local eddies are associated with shear mixing at the edge of thermals or the breakdown of large eddies through the turbulence cascading processes.

Schematics of the mean vertical profiles of potential temperature $\bar{\theta}(z)(\mathrm{K})$ and vertical heat fluxes $\overline{w^{\prime} \theta^{\prime}}(z)$ $\left(\mathrm{K} \mathrm{m} \mathrm{s}^{-1}\right)$ in a CBL are presented in the right panel of Fig. 1, where the overline is an ensemble average operator, ${ }^{1}$ and primes represent perturbations from the

\footnotetext{
${ }^{1}$ In this study, horizontal and time averaging are performed instead of ensemble averaging. The two are equivalent when the size of the dominant eddy motions, which is the boundary layer depth for the $\mathrm{CBL}$, is much less than the horizontal size of the domain (Wyngaard 2004).
} 


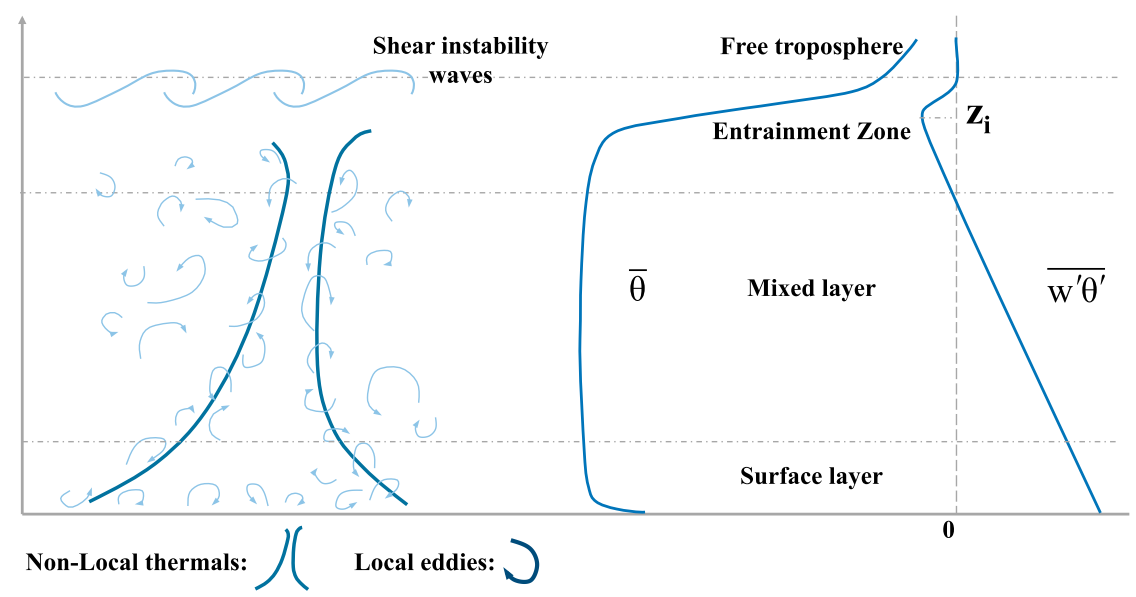

FIG. 1. (left) Schematic of the nonlocal thermals and local eddies, as well as (right) the potential temperature and heat flux profiles of the convective boundary layer.

ensemble mean. The $\bar{\theta}(z)$ profile outlines the stability of the CBL at different heights. The surface layer is characterized by a superadiabatic lapse rate, while the entrainment zone on top is stably stratified. In the nearneutral mixed layer in between, $\partial \bar{\theta}(z) / \partial z$ is slightly negative below $\sim 0.4 z_{i}$ and positive above (also shown later in Fig. 2a), where $z_{i}$ is the CBL depth (m), diagnosed as the vertical level of the minimum heat flux. The term $\overline{w^{\prime} \theta^{\prime}}(z)$ is linear from the surface up to the entrainment zone. This can be derived under horizontally homogeneous conditions and by assuming a quasisteady state such that $\partial \bar{\theta}(z) / \partial z$ is independent of time (Wyngaard 2010, chapter 11). Given the vertical profiles of $\bar{\theta}(z)$ and $\overline{w^{\prime} \theta^{\prime}}(z)$, an apparent "countergradient" region is identified in the upper part of the CBL, where the positive heat flux appears to be directed $u p$ the slightly positive local potential temperature gradient. The locally countergradient fluxes are associated with nonlocal thermals that span the depth of the CBL. At the thermal scale, the relevant vertical potential temperature gradient is related to the difference between $\bar{\theta}(z)$ and surface potential temperature $\bar{\theta}_{s}$, which is negative in the mixed layer [i.e., $\bar{\theta}(z)-\bar{\theta}_{s}<0$ ]. Therefore, from the perspective of thermals, warm surface air is transported upward to an elevation $z$ in the mixed layer where the potential temperature is slightly colder. Downgradient behavior of heat fluxes is then restored at the thermal scale.

Because of the presence of the locally countergradient fluxes associated with thermals, the classic gradient diffusion or $K$ theory for small-eddy turbulent fluxes,

$$
\overline{w^{\prime} \theta^{\prime}}=-K_{H} \frac{\partial \bar{\theta}}{\partial z}
$$

fails to represent vertical heat fluxes in the CBL. In Eq. (1), $w$ is the vertical velocity component $\left(\mathrm{m} \mathrm{s}^{-1}\right)$, and
$K_{H}$ is the eddy diffusivity for heat $\left(\mathrm{m}^{2} \mathrm{~s}^{-1}\right)$. Equation (1) is also known as the "small-eddy closure" that represents the downgradient turbulent diffusion by small eddies (Stull 1988, chapter 6). To account for the effects of nonlocal mixing in a scheme based on $K$ theory [Eq. (1)], an additional correction term $\gamma_{\mathrm{cg}}\left(\mathrm{K} \mathrm{m}^{-1}\right)$ is introduced alongside the gradient term $\partial \bar{\theta} / \partial z$ (Ertel 1942; Priestley and Swinbank 1947; Deardorff 1966):

$$
\overline{w^{\prime} \theta^{\prime}}=-K_{H}\left(\frac{\partial \bar{\theta}}{\partial z}-\gamma_{\mathrm{cg}}\right) \text {. }
$$

In Eq. (2), both $K_{H}$ and $\gamma_{\mathrm{cg}}$ are positive. Their product is meant to account for the difference between the total heat flux $\overline{w^{\prime} \theta^{\prime}}$ and the portion that is captured by the downgradient flux $-K_{H} \partial \bar{\theta} / \partial z$. Because $-\gamma_{\mathrm{cg}}$ has the opposite sign to the local gradient $\partial \bar{\theta} / \partial z$ in the upper part of the CBL, it is often referred to as the countergradient correction term or simply the countergradient term. In the lower part of the CBL, $-\gamma_{\mathrm{cg}}$ has the same sign as $\partial \bar{\theta} / \partial z$, so it acts as a downgradient term. In practice, the countergradient correction flux usually dominates over gradient flux in the mixed layer, while the latter contributes significantly in the surface layer and the entrainment zone where vertical gradients are large.

With the inclusion of $\gamma_{\mathrm{cg}}$, Eq. (2) forms the basis of a class of planetary boundary layer (PBL) schemes that represents $\mathrm{CBL}$ mixing in numerical weather prediction and global circulation models (e.g., Troen and Mahrt 1986; Bougeault and Lacarrere 1989; Zilitinkevich et al. 1999; Hong et al. 2006). Each individual scheme is distinguished by different formulations of $K_{H}$ and $\gamma_{\mathrm{cg}}$, which are usually derived based on the budget analysis of the prognostic equation for $\partial \overline{w^{\prime} \theta^{\prime}} / \partial t$ (e.g., Deardorff 1972; Therry and Lacarrère 1983; Holtslag and Moeng 1991). Note that only heat fluxes are examined in this 

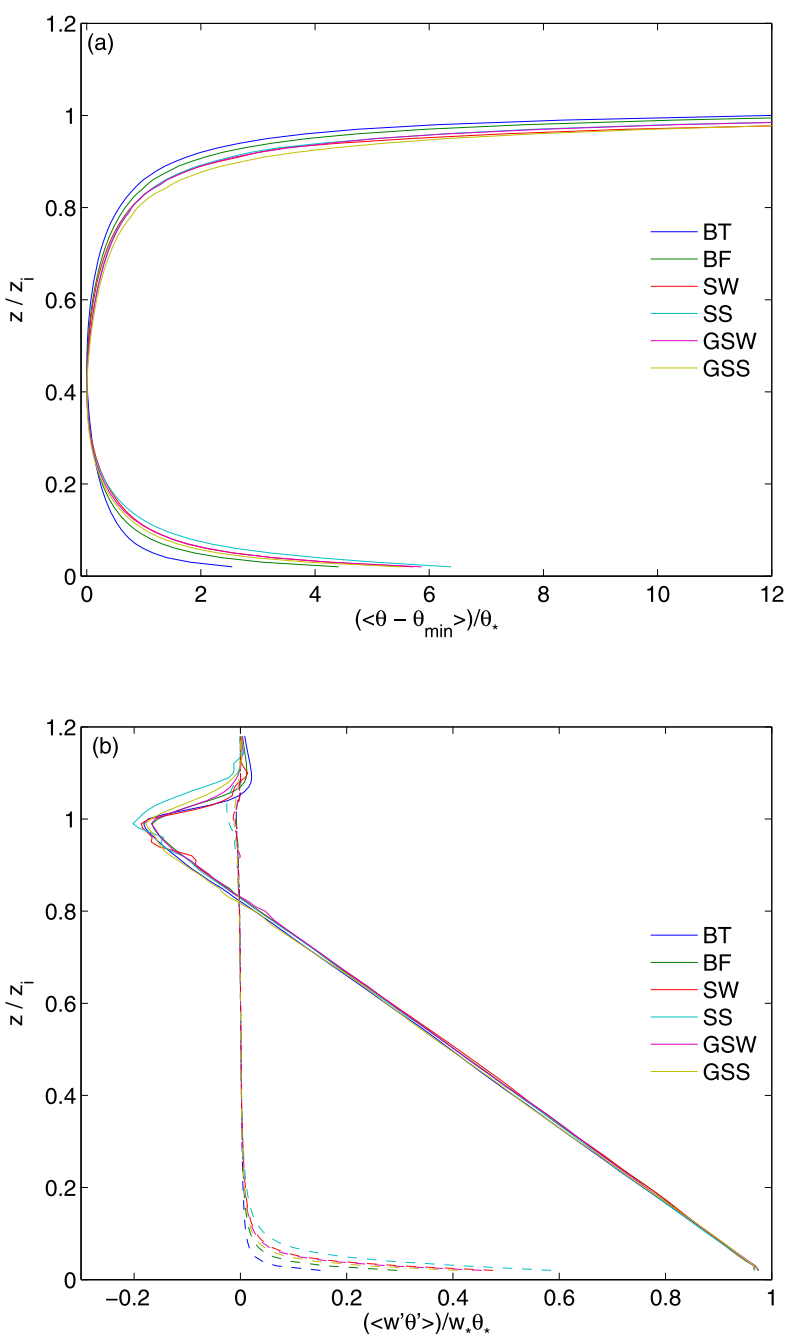

FIG. 2. Vertical profiles of the horizontally averaged and timeaveraged (a) potential temperature and (b) total heat flux (solid lines) and subgrid-scale heat flux (dashed lines) from the six cases. Note that $\langle\theta\rangle$ is normalized by subtracting $\langle\theta\rangle_{\min }$ and then dividing by $\theta_{*}$. The heat fluxes are normalized by the surface heat flux. Angle brackets indicate horizontal averaging.

study. Although some PBL schemes do include countergradient momentum and scalar correction terms (Hong et al. 2006; Sorbjan 2009), the presence of locally countergradient momentum, moisture, and other scalar fluxes have not been formally established, due partly to the lack of reliable field observations (Frech and Mahrt 1995; Brown and Grant 1997).

Although PBL schemes formulated based on Eq. (2) are both simple in design and effective in performance, their physical representation of CBL mixing has not been formally examined. Specifically, what physical processes do the gradient diffusion and the countergradient correction terms on the right-hand side of Eq. (2) represent? One convenient and almost straightforward interpretation is to connect the countergradient correction term $K_{H} \gamma_{\mathrm{cg}}$ to the nonlocal flux carried by the large organized thermals, ${ }^{2}$ and the gradient term $-K_{H} \partial \bar{\theta} / \partial z$ to the local flux associated with small eddies. Simply put, it is often explicitly or implicitly assumed that gradient and countergradient (GCG) terms represent local and nonlocal (LNL) fluxes, respectively. This study examines and eventually rebuts this hypothesis. The reason why this study is conducted is because, as well laid out by Plant and Yano (2015), parameterizations in general should be viewed as a fundamental science problem that demands physical insights, rather than a purely technical problem that can be tuned and manipulated in a piecemeal fashion. Inquiry into the physical insights of the GCG-based PBL schemes serves to expose the physical deficiency of these schemes, and deepen the understanding of CBL mixing processes on a fundamental level. Hopefully, these new insights will pave the way for the development of better PBL schemes.

To conduct the evaluation, we first perform six highresolution large-eddy simulations (LESs) of the CBL with different surface heating and vertical wind shear (see section 2). The GCG fluxes from two representative PBL schemes are then computed a priori from horizontally averaged LES data in section 3. In the meantime, with conditional sampling (CS) and Fourier decomposition of the LES flow field, heat fluxes associated with LNL eddies are derived. Several CS methods are adopted to reach a robust qualitative conclusion. By comparing the local (nonlocal) flux with its gradient (countergradient) counterpart, the physical representation of GCG-based PBL schemes is discussed in section 4. Overall conclusions are summarized in section 5.

\section{Methods}

\section{a. Case description and numerical setup}

LESs of six idealized CBL cases are performed with different surface heating and vertical wind shear. A list

\footnotetext{
${ }^{2}$ For example, in the literature, we often see statements such as "the term $K_{H} \gamma=\overline{w^{\prime} \theta^{\prime}}$ NL represents the non-local (NL) component of the heat flux" (Ghannam et al. 2017, p. 495), " $\gamma$ is the countergradient correction term, which incorporates the contribution of the large-scale eddies to the total flux" (Gibbs et al. 2011, p. 24320), "even more complex turbulence parameterizations include a nonlocal 'countergradient' mixing term to account for transport by the largest eddies in the opposite direction of local gradients" (Green and Zhang 2015, p. 1744), and "a solution was developed to represent the non-local effect of the large eddies by adding a countergradient term to the $K$ theory" (Pergaud et al. 2009, p. 84). While the authors meant well, to an uninformed reader these statements could be misinterpreted as direct implications that the countergradient correction term represents nonlocal flux.
} 
TABLE 1. List of model parameters and some statistics averaged from the last $2 \mathrm{~h}$ of the simulations. The unit for $z$ is meters.

\begin{tabular}{lcccc}
\hline \hline Run name & $\overline{w^{\prime} \theta^{\prime}}\left(\mathrm{K} \mathrm{m}^{-1}\right)$ & $U_{g}\left(\mathrm{~m} \mathrm{~s}^{-1}\right)$ & $-z_{i} / L$ & Organization \\
\hline BT & 0.20 & 0 & $2.12 \times 105$ & Cell \\
BF & 0.20 & 10 & 20.7 & Transition \\
SW & 0.05 & 10 & 6.27 & Roll \\
SS & 0.05 & 15 & 2.65 & Roll \\
GSW & 0.05 & $0.002 z+8$ & 5.57 & Roll \\
GSS & 0.05 & $0.005 z+5$ & 8.00 & Roll \\
\hline
\end{tabular}

of model parameters is presented in Table 1 . The first four cases feature barotropic conditions. The model setup follows Shin and Hong (2013) with prescribed surface heating and pressure gradient forcings. Among the four cases, a constant heat flux of $0.20 \mathrm{~K} \mathrm{~m}^{-1} \mathrm{~s}^{-1}$ is imposed on the two buoyancy-driven cases, called BT and BF. In the absence of geostrophic winds, organized convective cells develop in the BT case. In addition to strong surface heating, the BF case is also driven by a constant $10 \mathrm{~m} \mathrm{~s}^{-1}$ geostrophic wind. The convective structures in the BF case are not well defined. The parameter $-z_{i} / L$ indicates the type of organized convection. Here, $L=-u_{*}^{3} \theta_{0} /\left(\kappa g w^{\prime} \theta_{s}^{\prime}\right)$ is the Obukhov length $(\mathrm{m})$, $u_{*}$ is the surface friction velocity $\left(\mathrm{m} \mathrm{s}^{-1}\right), \theta_{0}$ is the reference potential temperature $(\mathrm{K}), \kappa(=0.4)$ is the von Kármán constant, $g$ is the gravitational acceleration constant $\left(\mathrm{m} \mathrm{s}^{-2}\right)$, and $\overline{w^{\prime} \theta^{\prime}}$ is the surface heat flux. The value of $-z_{i} / L$ for the BF case is about 20 , which falls into the transition range $\left(-z_{i} / L \approx 15-20\right)$ where the organized convection changes from cellular (large $-z_{i} / L$ ) to roll (small $\left.-z_{i} / L\right)$ formations (Salesky et al. 2017). The weak-shear (SW) and strong-shear (SS) cases are conducted with a smaller surface heat flux $\left(0.05 \mathrm{~K} \mathrm{~m}^{-1} \mathrm{~s}^{-1}\right)$ compared to the buoyancy-driven cases. The imposed geostrophic winds are 10 and $15 \mathrm{~m} \mathrm{~s}^{-1}$ for the SW and SS cases, respectively. With less heating, horizontal roll vortices form in the presence of shear in both cases. In addition to the four barotropic cases, two more baroclinic cases are performed with linear geostrophic shear (GS). The GSW and GSS cases have 2 and $5 \mathrm{~m} \mathrm{~s}^{-1} \mathrm{~km}^{-1}$ shear. The geostrophic wind speeds for both GS cases are $10 \mathrm{~m} \mathrm{~s}^{-1}$ at 1-km height, same as the SW case. All runs are initialized with a potential temperature profile following Shin and Hong (2013), where

$$
\theta= \begin{cases}300 \mathrm{~K}, & 0<z \leq 925 \mathrm{~m} \\ 300 \mathrm{~K}+(z-925 \mathrm{~m}) \times 0.0536 \mathrm{~K} \mathrm{~m}^{-1}, & 925<z \leq 1075 \mathrm{~m} \\ 308.05 \mathrm{~K}+(z-1075 \mathrm{~m}) \times 0.003 \mathrm{~K} \mathrm{~m}^{-1}, & z>1075 \mathrm{~m}\end{cases}
$$

The initial $\theta(z)$ has a sharp inversion between 925 and $1075 \mathrm{~m}$, which effectively caps the growth of the CBL throughout the entire simulation. The initial wind profile is geostrophic. The Coriolis parameter is set to $10^{-4} \mathrm{~s}^{-1}$. The surface roughness length is $0.1 \mathrm{~m}$.

The LESs are performed on a doubly periodic domain of $(12 \mathrm{~km}, 12 \mathrm{~km}, 2 \mathrm{~km})$, with Rayleigh damping applied to the top $500 \mathrm{~m}$. The horizontal domain size is large compared to the CBL depth. This allows inclusion of a relatively large number of samples, which are the organized convective eddies, for horizontal averaging to minimize uncertainties of the estimated mean. We adopt $25-\mathrm{m}$ horizontal and $12.5-\mathrm{m}$ vertical grid spacings, so that the CBL is well resolved with a negligible contribution from the subgrid scale (SGS), except for the first few grid points above the ground. The 1.5-order turbulent kinetic energy (TKE) closure of Moeng (1984) is adopted for SGS turbulence. Based on Sullivan and Patton (2011), the CBL is highly resolved (i.e., converges to extremely high-resolution simulations) when the boundary layer depth to grid spacing ratio $z_{i} / \Delta$ exceeds 60 . Under the current setup, $z_{i} / \Delta$ is approximately 50 for all cases (see Table 1), close to the highly resolved criterion. The CBL reaches a statistical quasisteady state after $6 \tau$, where $\tau \equiv z_{i} / w_{*}$ is the large-eddy turnover time, approximately 600 and $800 \mathrm{~s}$ for buoyancy and shear cases (Shin and Hong 2013), and $w_{*} \equiv\left(g / \theta_{0} \overline{w^{\prime} \theta^{\prime}}{ }_{s} z_{i}\right)^{1 / 3}$ is the convective velocity scale $\left(\mathrm{m} \mathrm{s}^{-1}\right)$. Therefore, the two buoyancy cases are run for $4 \mathrm{~h}$ whereas the shear cases are run for $5 \mathrm{~h}$. Data are output at every $10 \mathrm{~min}$, and a total of 13 snapshots from the last $2 \mathrm{~h}$ are used for the analyses. Results presented in this study are both horizontally averaged and time averaged, unless otherwise stated.

The Advanced Regional Prediction System (ARPS) is used for the simulations. ARPS was developed at the Center for Analysis and Prediction of Storms at the University of Oklahoma. It is a nonhydrostatic mesoscale and convective-scale finite-difference NWP model that is also suitable for LES (e.g., Chow et al. 2005). 
ARPS uses a generalized height-based terrain-following coordinate on an Arakawa C-grid. A mode-splitting time integration scheme is employed (Klemp and Wilhelmson 1978). More details on ARPS are documented in Xue et al. (2000, 2001).

\section{b. Conditional sampling methods}

Unfortunately, there are no unique and strict definitions of nonlocal and local eddies. Quite a few conditional sampling (CS) methods have been proposed in the literature to separate coherent nonlocal CBL thermals from the environment, using data from field observations (Warner and Telford 1967; Lenschow and Stephens 1980), wind tunnel measurements (Fedorovich and Kaiser 1998; Kaiser and Fedorovich 1998), and most often LESs (Siebesma et al. 2007; Couvreux et al. 2010; Shin and Hong 2013; Park et al. 2016). Each method is characterized with a unique set of CS criteria, which usually involves certain sampling parameters that have no strict optimal values (Shin and Hong 2015). For example, the method of Siebesma et al. (2007, hereafter SST07) identifies updrafts based on

$$
w(x, y, z)>w_{p}(z)
$$

where $p$ is a percentage chosen to be $1 \%, 3 \%$, and $5 \%$ in SST07 and $w_{p}(z)$ refers to the top $p$ of the vertical velocity $w$ at height $z$. After separation of nonlocal updrafts from the environment, the LNL fluxes are computed by

$$
\begin{aligned}
{\overline{w^{\prime} \theta^{\prime}}}_{\mathrm{NL}} & =p\left(w_{u}-\bar{w}\right)\left(\theta_{u}-\bar{\theta}\right)+(1-p)\left(w_{e}-\bar{w}\right)\left(\theta_{e}-\bar{\theta}\right) \\
{\overline{w^{\prime} \theta^{\prime}}}_{L} & =p{\overline{w^{\prime \prime} \theta^{\prime \prime}}}^{u}+(1-p) \overline{w^{\prime \prime} \theta^{\prime \prime}}
\end{aligned}
$$

where subscript and superscript $u$ and $e$ refer to horizontally averaged updrafts and environment, respectively. Double primes denote perturbations from the local environment, for example, within the updraft $w^{\prime \prime}=w-w_{u}$. In the idealized simulations conducted in this study, $\bar{w}$ is zero in Eq. (5). The terms on the righthand side of Eq. (5) represent advective-like thermal and environment transport, while those of Eq. (6) represent subthermal and subenvironmental fluxes.

This study adopts the method of SST07 for its simplicity. The parameter space of $p$ in Eq. (4) is thoroughly explored. In addition, two other more sophisticated CS methods proposed by Couvreux et al. (2010) and Park et al. (2016) are also tested for the BT case. As shown later in section $3 \mathrm{~b}$ and appendix B, qualitatively similar features emerge in spite of the arbitrary choices of the free parameter as well as the different CS methods used.
These shared qualitative features describe the fundamental characteristics of the LNL eddies, which is the focus of this study.

\section{Results}

\section{a. Gradient and countergradient fluxes}

The vertical profiles of normalized potential temperature $\Theta$ as a function of the normalized height $Z=z / z_{i}$ are presented in Fig. 2a. The value of $\Theta(Z)$ is obtained by first subtracting the minimum value $\langle\theta\rangle_{\text {min }}$ from $\langle\theta\rangle(z)$, where the angle brackets represent horizontal averaging, and then dividing by the convective temperature scale $\theta_{*} \equiv \overline{w^{\prime} \theta^{\prime}} / w_{*}$. Differences in the profiles of the six cases are mainly observed in the surface layer. The free-convection case (BT) has the least negative surface-layer gradient. With the addition of vertical wind shear and/or the reduction of surface heat fluxes, the surface layer becomes more superadiabatic. Above the surface layer, $\Theta(Z)$ values from all six cases overlap well in the mixed layer, while some differences are observed in the entrainment zone. Since we are most interested in the mixed layer where nonlocal thermals are most energetic and countergradient fluxes are found, and given the universal collapse of $\Theta(Z)$ in the mixed layer, we do not distinguish $\Theta(Z)$ among different cases hereafter.

Despite the implication of vanishing gradients by the name "mixed layer," $\partial \Theta / \partial Z$ is nonzero throughout the depth of the CBL. Note that $\Theta(Z)$ has a global minimum slightly above $0.4 z_{i}$, where $\partial \Theta / \partial Z$ is negative below and positive above. The gradient sharpens toward the ground due to surface heating, and rapidly increases toward the overcapping inversion approaching the CBL top. The profile of $\Theta(Z)$ and the $\sim 0.4 z_{i}$ location for the zero-crossing of $\partial \Theta / \partial Z$ have been documented by many independent studies (e.g., Holtslag and Moeng 1991; Stevens 2000; SST07). A direct implication from such vertical profiles of $\Theta(Z)$ for the flux parameterization is that the gradient diffusion term in Eq. (2) must be positive below $\sim 0.4 z_{i}$ and negative above.

The vertical profiles of normalized heat fluxes $\left\langle\overline{w^{\prime} \theta^{\prime}}\right\rangle / w_{*} \theta_{*}$ from all six cases collapse onto a universal curve shown in Fig. 2b. The heat flux profiles are linear up to the boundary layer top, with zero-crossings occurring slightly above $0.8 z_{i}$. The magnitudes of the minimum heat fluxes in the entrainment zone are about $18 \%$ of their surface values. Also presented in Fig. $2 b$ are the SGS heat fluxes from the turbulence closure. The SGS fluxes are almost zero everywhere except in the surface layer below $\sim 0.1 z_{i}$. This suggests that the 
simulated CBLs are well resolved with the current model setup described in section 2.

The Holtslag and Moeng (1991) (HM) and Yonsei University (YSU) (Hong et al. 2006) schemes are chosen to represent the class of gradient and countergradient correction-based PBL schemes. The HM scheme is selected because its countergradient term is formulated based on LES statistics and is therefore considered accurate. The YSU scheme is selected because it is a widely used PBL scheme with good performance. Their formulations are presented in appendix A. Although only two representative schemes are examined, the qualitative behavior of their GCG terms is not limited to these two particular schemes and should apply to GCG-based PBL schemes in general.

Figure 3 presents the partition of heat fluxes into the GCG components for the two PBL schemes. Note that the YSU scheme includes an entrainment term in addition to the GCG terms. Aside from horizontal and time averaging, all normalized fluxes are also averaged among the six cases. (For individual flux profiles of each case, see Fig. A1 in appendix A.) Case averaging is applied to the normalized total heat flux and the countergradient flux profiles because they overlap nicely among the six cases (see Figs. $2 b$ and A1). The normalized gradient flux profiles shown in the appendix (Fig. A1) exhibit casewise differences mostly below $\sim 0.4 z_{i}$. This is likely attributable to the effects of wind shear, which is most prominent in the surface layer. Since case averaging does not alter the general conclusions about gradient fluxes in this study, it is also applied to the gradient fluxes in Fig. 3 for clarity of presentation.

Another thing to note is that the a priori computed GCG fluxes do not necessarily add up to the total flux. This is reflected by comparing the directly computed gradient flux and the residual flux in Fig. 3. The latter is obtained by subtracting the nongradient flux from the total flux. The difference between the directly and indirectly computed (i.e., the residual) gradient fluxes is due to the difference between the a posteriori profiles produced by a PBL scheme and the a priori profiles averaged from LES data. Specifically, $\bar{\theta}(z)$ obtained from a single-column model using a PBL scheme usually differs from LES obtained $\langle\theta\rangle(z)$. Examples can be found in Fig. 10 of Svensson et al. (2011) and Fig. 2 of Wang et al. (2016). When a PBL scheme is applied to model CBL mixing, the predicted heat flux profile tends to be linear as presented in the schematic in Fig. 1b, as long as sufficient amount of mixing is provided by the PBL scheme. When further constrained by boundary conditions (i.e., the surface heat flux), a posteriori heat flux profiles from different PBL schemes collapses onto a universal curve. This is demonstrated in
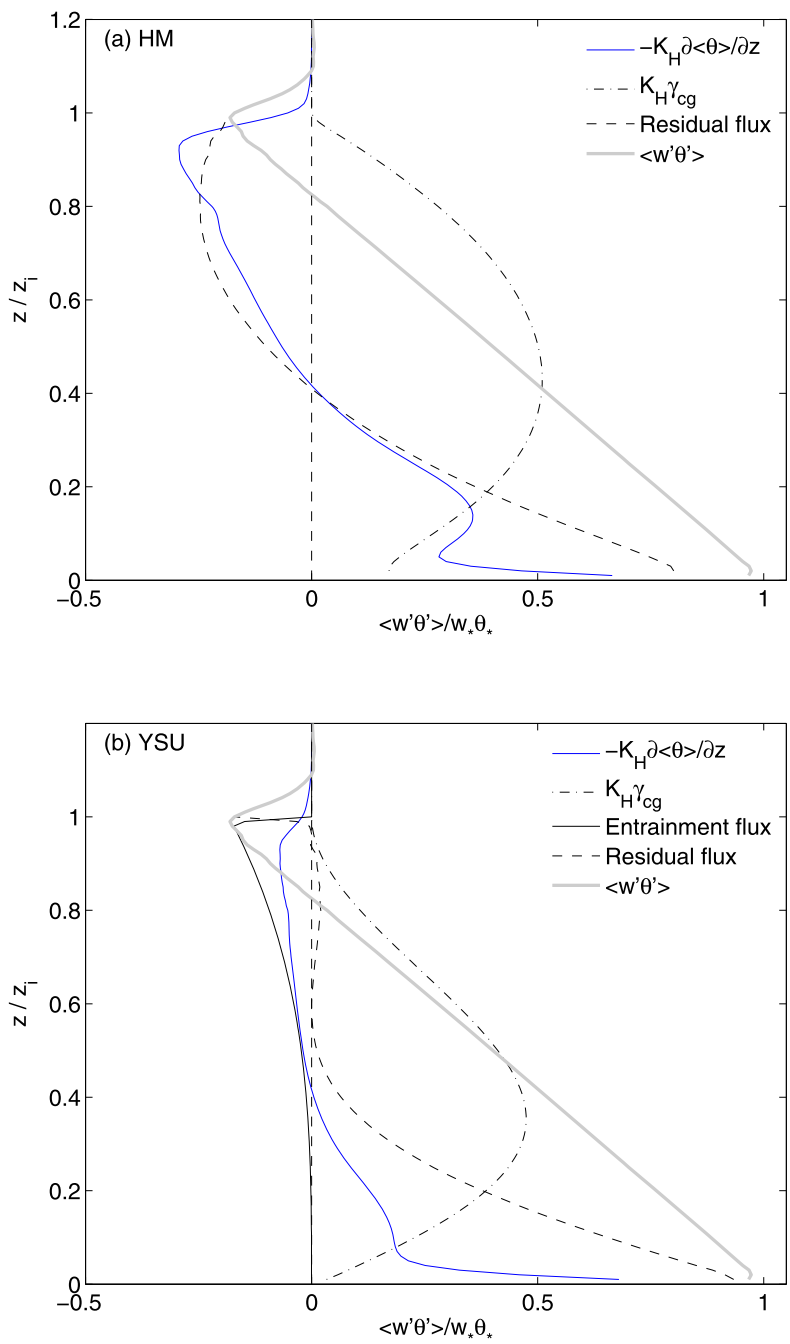

FIG. 3. Vertical profiles of the horizontally and time-averaged heat flux partitions for (a) the HM scheme and (b) the YSU scheme. All fluxes are averaged among the six cases. The gradient fluxes $-K \partial\langle\theta\rangle / \partial z$ include the SGS fluxes. The vertical dashed lines represent zero flux.

Wang et al. (2016), where 11 PBL schemes are evaluated on an idealized CBL case. The heat flux profiles within the CBL exhibit extreme insensitivity to the choice of the PBL scheme (see their Figs. 4a and 4b), even though the eddy diffusivity profiles differ widely among schemes (see their Figs. 3a-d). As the total heat flux is strongly constrained, the potential temperature profile is then directly related to the profiles of $K_{H}$ and $\gamma_{\mathrm{cg}}$ of a particular PBL scheme as dictated by Eq. (2), and therefore exhibits scheme-wise variations. Similarly, in a priori evaluation where the potential temperature profiles obtained from horizontally averaged LES data are fixed (see Fig. 2a), different PBL schemes with different formulations of $K_{H}$ and $\gamma_{\mathrm{cg}}$ can also predict heat flux profiles that differ from the LES-averaged profile in Fig. 3. In this study, we are 
only interested in the qualitative behavior of the GCG fluxes. Differences between the directly computed gradient fluxes and the residual fluxes do not affect the discussions and conclusions presented below.

In Fig. 3a, gradient flux of the HM scheme is dominant in the surface layer and the entrainment zone. In the mixed layer between $0.2 z_{i}$ and $0.8 z_{i}$, contributions from the countergradient flux are more substantial. The gradient flux switches direction at approximately $0.4 z_{i}$ as a result of the vertical gradient reversal at this elevation (see Fig. 2). Above $\sim 0.4 z_{i}$, gradient flux contributes negatively to an overall positive heat flux. Its negative contribution is counteracted by the countergradient flux, which is larger than the total heat flux. A similar profile is given in Fig. 11 of SST07, who used Cuijpers and Holtslag's (1998) PBL scheme for $K_{H}$ and $\gamma_{\mathrm{cg}}$.

The vertical heat flux profile produced by the YSU scheme is presented in Fig. 3b. Behavior of the GCG fluxes is qualitatively similar to that from the HM scheme. The directly computed YSU gradient flux also switches direction at around $0.4 z_{i}$. However, its contribution to the total flux is much smaller compared to that of the HM scheme. The countergradient flux of the YSU scheme has a similar parabolic shape as that of the HM scheme. Above $\sim 0.5 z_{i}$, its magnitude exceeds that of the total flux. Apart from the GCG terms, the YSU scheme has an entrainment term that is set by a diagnosed negative entrainment flux at the CBL top, and decays downward as $\left(z / z_{i}\right)^{3}$ [see Eq. (A3)]. This term is an explicit representation of entraining warmer (potential temperature-wise) free-tropospheric air into the boundary layer, which is also considered as a nonlocal mixing process (Noh et al. 2003; Hong et al. 2006). For many PBL schemes under the GCG framework such as the HM scheme presented in Fig. 3a, the representation of entrainment flux relies on the gradient diffusion term alone. The presence of the explicitly parameterized entrainment flux in the YSU scheme explains the smaller (i.e., less negative) gradient flux in the upper half of the CBL. It also accounts for the upward shift of the location where the countergradient flux exceeds the total flux from around $0.4 z_{i}$ to $0.5 z_{i}$.

\section{b. Local and nonlocal fluxes}

The horizontally and time- and case-averaged updraft and environment profiles of the potential temperature and vertical velocity are presented in Fig. 4 . The profiles are obtained using the SST07 method with $p=10 \%$ following Shin and Hong (2013). In Fig. 4a, thermals are much warmer than the horizontally averaged potential temperature $\langle\theta\rangle$ up to around $0.8 z_{i}$. In the entrainment zone, penetrating thermals are colder than $\langle\theta\rangle$. The mean potential temperature of the environment $\left\langle\theta_{e}\right\rangle$ is only slightly colder than $\langle\theta\rangle$ throughout the depth of the CBL. For the vertical velocity, the updraft-mean $\left\langle w_{u}\right\rangle$ is roughly $1.5 w_{*}$ in the mixed layer, although this value is sensitive to the choice of $p$. The environment is associated with a negative velocity $\left\langle w_{e}\right\rangle$ of much smaller magnitude than $\left\langle w_{u}\right\rangle$, indicating slowly subsiding environmental air.

The LNL partition of heat flux with $p=10 \%$ is presented in Fig. 5a. Fluxes computed from Eq. (6) and the SGS fluxes presented in Fig. $2 b$ are combined to form the local fluxes in this panel. The LNL fluxes from all six cases agree well throughout the depth of the CBL especially in the mixed layer, although some small differences are observed in the surface layer below $0.1 z_{i}$ and in the entrainment layer above $0.8 z_{i}$. Both LNL heat fluxes are directed upward over the depth of the mixed layer. The zero-crossings for the local and nonlocal fluxes are around 0.8 and $0.9 z_{i}$, respectively. For most of the entrainment zone, both fluxes are directed downward.

The computed LNL fluxes depend on the choice of the parameter $p$ in the SST07 method. To investigate the variability of the flux partitions, flux profiles for $p$ ranging from $1 \%$ to $50 \%$ are computed at $1 \%$ intervals and five of them are presented in Figs. 6a and 6b. Higher percentage values are not tested since observations (Lenschow and Stephens 1980) and simulations (Couvreux et al. 2010) both suggest that the areal fraction of organized nonlocal structures do not exceed $50 \%$. The LNL fluxes are also case-averaged for clarity of presentation. Comparing Figs. $6 \mathrm{a}$ and 6b, the nonlocal fluxes are in general larger than their respective local counterparts in terms of magnitude. This is similar to what other studies have found (Couvreux et al. 2010; Shin and Hong 2015). The nonlocal flux contribution to the total flux is nonlinear with respect to $p$ in Fig. 6a, and likewise for the local flux contribution in Fig. 6b. At small $p$, nonlocal fluxes increase with $p$ and maximize when $p \approx 30 \%$. Afterward, contributions from nonlocal fluxes decrease with further increase in $p$. Despite the quantitative differences in flux partitions, the qualitative behavior of $p$ ranging from $1 \%$ to $50 \%$ remains the same: in the mixed layer where the total flux is positive, both LNL fluxes contribute positively; in the entrainment zone where the total flux is negative, both LNL fluxes contribute negatively. The zero-crossings for both the nonlocal and local fluxes are close to $0.8 z_{i}$ for all $p$ values evaluated (results not shown). Also note that when $p=15 \%$, the resulting heat flux partition based on SST07 is closest to that from the more sophisticated Couvreux et al. (2010) method presented in appendix B.

The LNL fluxes can be further separated into updraft and environment contributions according to Eqs. (5) and (6). The results are presented in Figs. $6 \mathrm{c}$ and $6 \mathrm{~d}$. For nonlocal fluxes, those associated with thermals dominate over those with the environment in Fig. 6c. Note 

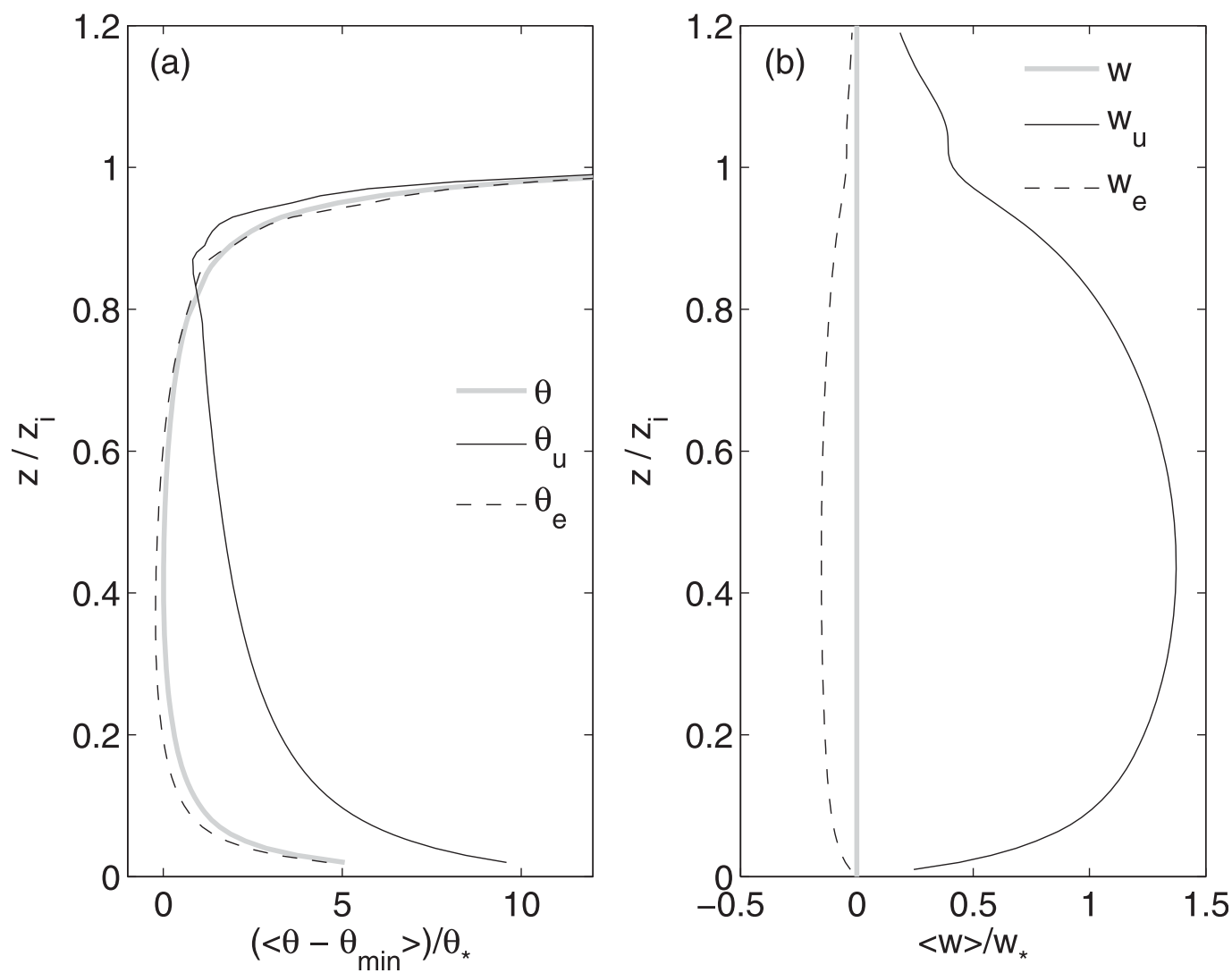

FIG. 4. Vertical profiles of the horizontally and time- and case-averaged (a) potential temperature and (b) vertical velocity based on the CS method of SST07 with $p=10 \%$. Black solid and dashed lines represent averages of the updraft and the environment, respectively. Gray solid lines represent horizontally averaged profiles. Here $\langle\theta\rangle$ is normalized by subtracting $\langle\theta\rangle_{\min }$ and then dividing by $\theta_{*}$, and $w$ is normalized by $w_{*}$.

that the nonlocal environment fluxes, representative of organized subsidence, are all positive below $\sim 0.8 z_{i}$ for the range of $p$ values tested. This is in contradiction to the explicitly parameterized negative entrainment flux of the YSU scheme in Fig. 3b, although the vertical divergences (gradients) of both the diagnosed and parameterized fluxes have the same signs, indicating warming of the CBL. For the local heat flux component in Fig. 6 d, when $p$ is less than $30 \%$, local eddies associated with subenvironmental variations produce stronger fluxes than those due to subthermal variations. Beyond $p=30 \%$, contributions from updraft-associated local eddies dominate.

Unlike the parameterized gradient fluxes in Fig. 3, the LES diagnosed local fluxes in Figs. $5 \mathrm{a}$ and $6 \mathrm{~b}$ do not have any negative values between $\sim 0.4 z_{i}$ and $\sim 0.8 z_{i}$, where $\partial\langle\theta\rangle / \partial z$ is positive (see Fig. 2a). As a consequence, the magnitude of the diagnosed nonlocal flux is smaller than that of the net flux throughout the depth of the CBL (Figs. 5a and 6a). In contrast, the magnitude of the countergradient correction flux exceeds that of the net flux in the upper mixed layer (Fig. 3). The above conclusion is robust for this particular CS method since the parameter space of the method's only free parameter $p$ is quite thoroughly explored. But even so, these conclusions could still be undermined by the objectiveness of the method itself and be considered methoddependent. Therefore, in appendix B, two sophisticated CS methods by Couvreux et al. (2010) and Park et al. (2016) are also tested. Both yield qualitatively similar results as the SST07 method.

For a more subjective approach, the Fourier decomposition is adopted to investigate the possible existence of downward-directed local flux as well as larger-than-total nonlocal flux in upper mixed layer. The LES flow field is projected into a wavenumber space through horizontal Fourier transform. The heat flux cospectra $F_{w \theta}$ are computed at different vertical elevations and presented in Fig. 7. The Fourier decomposition is not strictly a conditional sampling method that separates thermals from the environment. However, it does achieve a subjective separation of scales. The cospectral density $F_{w \theta}$ at wavenumber $k$ indicates the contribution from turbulent eddies of size $\lambda=2 \pi / k$ to the total flux. 

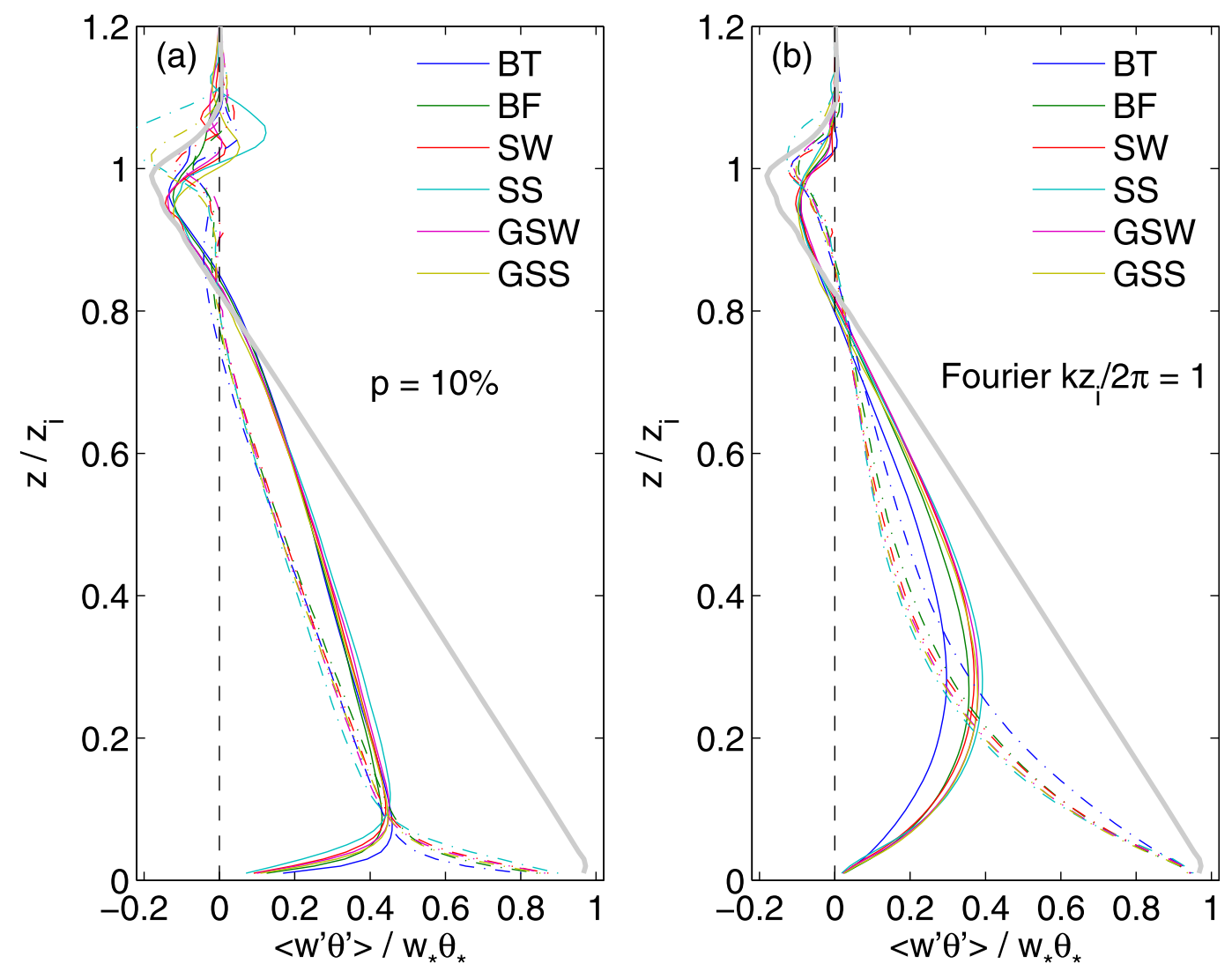

FIG. 5. Vertical profiles of the horizontally and time-averaged normalized heat flux and its partition into LNL components, by the (a) $p=10 \%$ criterion and (b) the Fourier transform criterion for $k z_{i} / 2 \pi=1$. Solid lines represent nonlocal fluxes and dashed lines represent the sum of local and SGS fluxes. The gray solid lines represent the total heat fluxes. The vertical dashed lines represent zero flux.

Here $k$ is the magnitude of the horizontal wavenumber vector $\left(k_{x}, k_{y}\right)$. The cospectra exhibit only small differences among the six cases tested (not shown), and therefore are case-averaged. Values of $F_{w \theta}$ at five selected elevations are presented in Fig. 7a. At $0.2 z_{i}, 0.4 z_{i}$, and $0.6 z_{i}, F_{w \theta}$ is positive for all wavenumbers. The largest $F_{w \theta}$ values at these three elevations are found at wavelengths between $1 z_{i}$ and $2 z_{i}$, corresponding to the size of the organized convective structures in the CBL. At $0.8 z_{i}, F_{w \theta}$ becomes negative within a limited range of small wavenumbers, indicating the effect of penetrating thermals into the entrainment layer. Meanwhile the small eddies at large wavenumbers still contribute positively to $F_{w \theta}$. At the CBL top $\left(1.0 z_{i}\right), F_{w \theta}$ becomes negative for all wavenumbers.

Contours of the normalized $F_{w \theta}$ as a function of wavenumber and height are presented in Fig. $7 \mathrm{~b}$, with the zero-flux contour line highlighted by the numeral 0 . Slightly below $0.8 z_{i}$, turbulent eddies of all sizes contribute positively to an overall positive heat flux. Similar behavior is found in the wind tunnel measurements of
Kaiser and Fedorovich (1998, their Fig. 8), where $F_{w \theta}$ is positive for all wavenumbers below $0.75 z_{i}$. In the entrainment zone above $0.8 z_{i}, F_{w \theta}$ is negative for almost all $k$, except for at the very high wavenumbers. The cause of these very small positive contributions are not clear, as they could be due to numerical errors toward the cutoff wavenumber. The only place where positive and negative $F_{w \theta}$ values coexist is found in a narrow region around $0.8 z_{i}$, although the magnitude of the net heat flux at this elevation range is close to zero (see Fig. 2b). Our results here are in contradiction to Ebert et al. (1989, their Fig. 22), where they showed that heat fluxes associated with medium-sized $\left(0.12-0.44 z_{i}\right)$ eddies switch sign from positive below to negative above $0.5 z_{i}$. The large $\left(0.44-1.50 z_{i}\right)$ eddies contribute positively to the heat flux throughout the depth of the CBL, while small $\left(0-0.12 z_{i}\right)$ eddies have negligible contribution from around $0.2 z_{i}$ to $0.8 z_{i}$. In our study, LES-resolved eddies of all sizes unanimously contribute positive heat fluxes below $\sim 0.8 z_{i}$. Eddy sizes in Ebert et al. (1989) were diagnosed from the LES-derived transilient matrix 

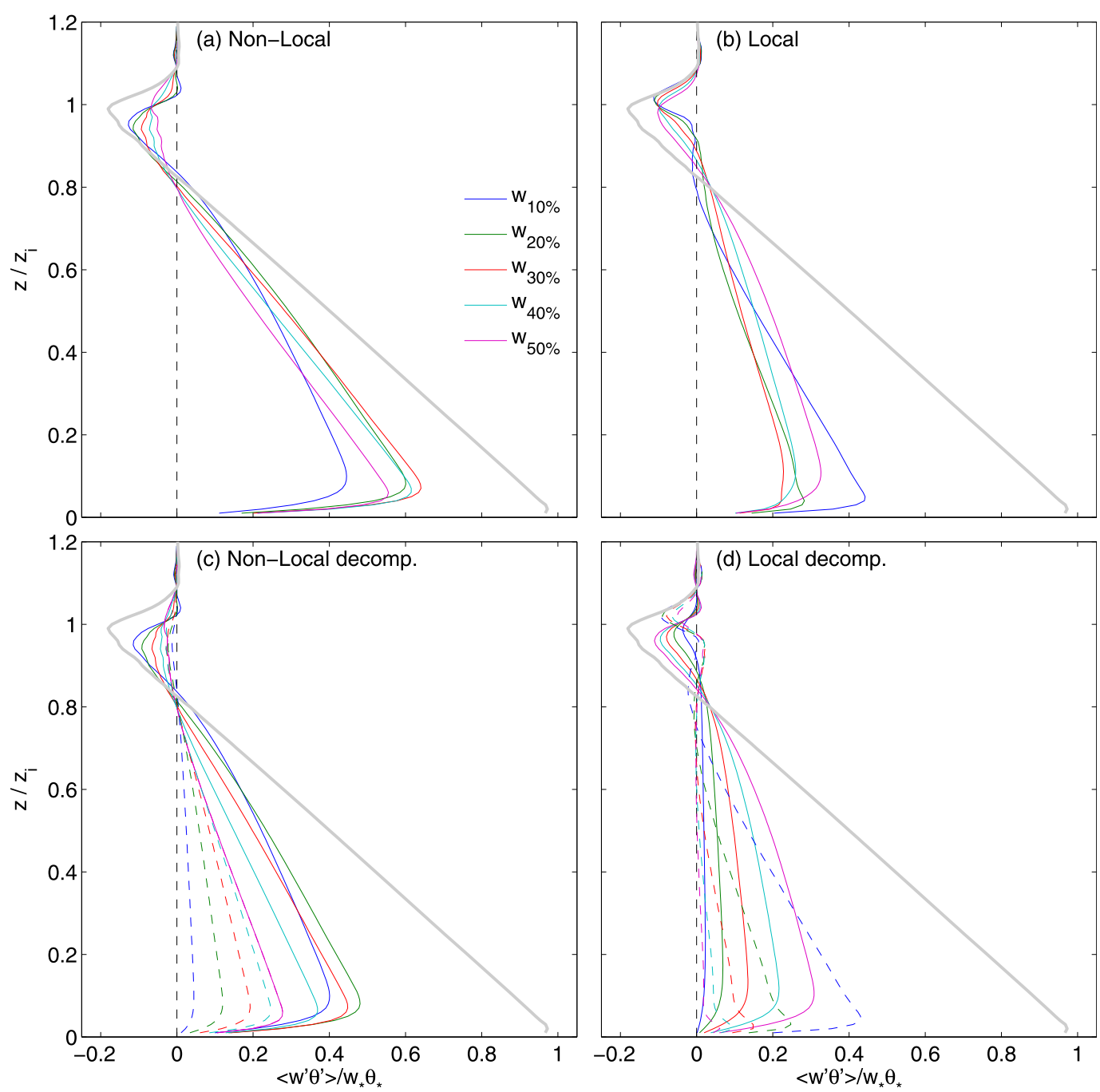

FIG. 6. Vertical profiles of the horizontally and time- and case-averaged normalized (a) nonlocal and (b) local heat fluxes for $p=10 \%, 20 \%, 30 \%, 40 \%$, and 50\%. (c),(d) The LNL fluxes are further decomposed into updraft (solid lines) and environmental (dashed lines) contributions.

(Stull 1993). The discrepancy could be caused by the relatively coarse resolution of their LES $(100-\mathrm{m}$ isotropic spacing). It is also likely that some wave motions are misdiagnosed as turbulent eddies. This is evident from the large magnitude of the individual heat fluxes far exceeding the magnitude of the net flux above $0.8 z_{i}$ in Ebert et al. (1989, their Fig. 22).

Although Fourier decomposition is not strictly a CS method, we could still objectively decide on a critical wavenumber $k_{c}$ to split between nonlocal and local eddies. Here, $k_{c}=2 \pi / z_{i}$ is selected because it is associated with the largest cospectral density $F_{w \theta}$ in Fig. 7a, corresponding to the most energetic eddies in transporting heat fluxes. Based on $k_{c}$, the local $\left[\int_{k_{c}}^{\infty} F_{w \theta}(k) d k\right]$ and nonlocal $\left[\int_{0}^{k_{c}} F_{w \theta}(k) d k\right]$ fluxes for all six cases are computed and presented in Fig. 5b. The differences among cases are quite small, except for the freeconvective (BT) case whose nonlocal (local) flux is somewhat smaller (larger) than the rest. In Fig. 5b, the zero-crossings of both LNL fluxes are nearly collocated around $0.8 z_{i}$. Note that toward the top of the entrainment layer (i.e., above $1.0 z_{i}$ ), local flux dominates. This is consistent with the direct numerical simulation results of Garcia and Mellado (2014), where a two-layer structure of the entrainment zone was found, with the upper layer dominated by local eddies generated by shear instability, and lower layer controlled by overshooting thermals. Different choices of $k_{c}$ produce different LNL flux partitions as shown in Fig. 8. Variations of the nonlocal and local fluxes with respect to $k_{c}$ are monotonic. The nonlocal flux converges to the total flux as $k_{c}$ increases, because an 

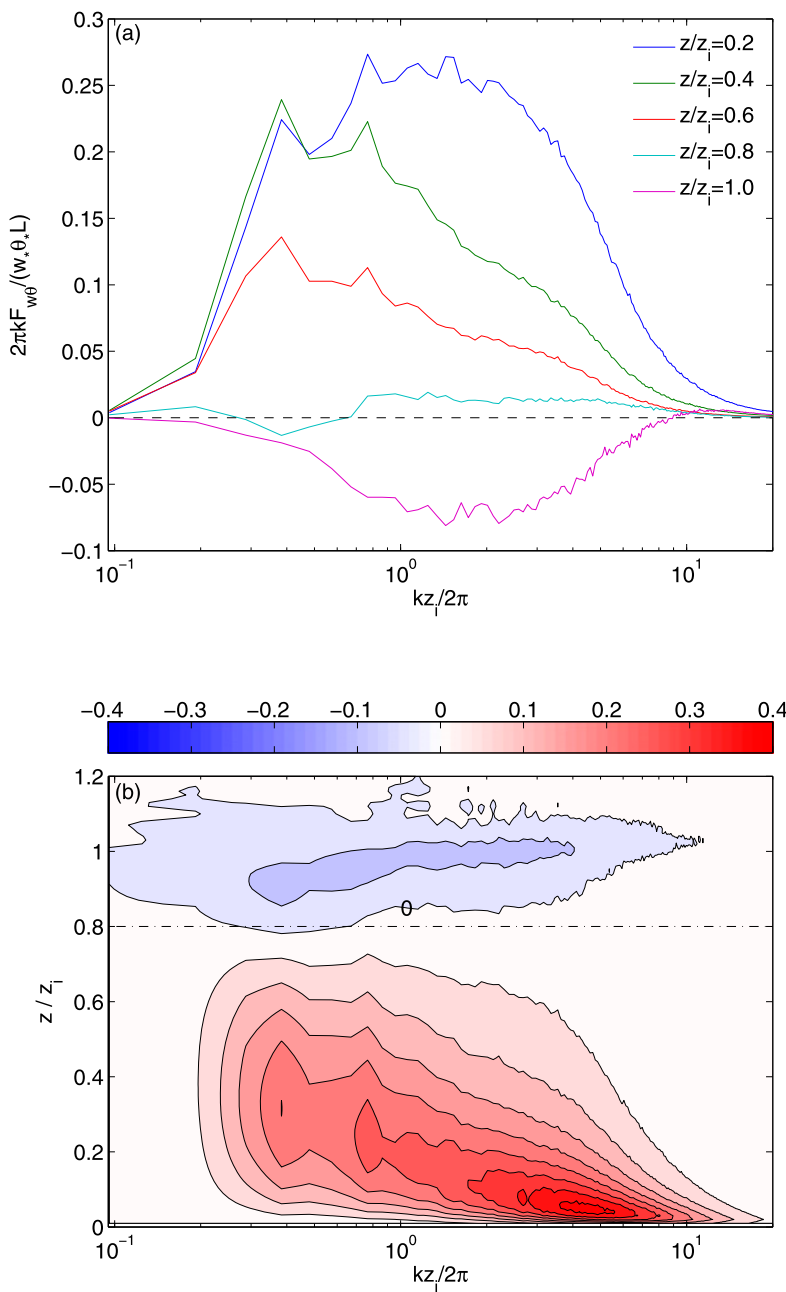

FIG. 7. (a) Normalized time- and case-averaged heat flux cospectra $2 \pi k F_{w \theta} /\left(w_{*} \theta_{*} L\right)$ at five selected heights within the CBL, where $L$ here is the domain width. (b) Contour plot of the normalized time- and case-averaged cospectra as a function of normalized wavenumber $k z_{i} / 2 \pi$, and normalized height $z / z_{i}$. The contour interval is 0.05 . The numeral 0 marks the zero contour line. The horizontal dash-dotted line marks the elevation of $0.8 z_{i}$.

increasing number of smaller eddies is counted as nonlocal ones. The zero-crossings of the LNL fluxes are also found around $0.8 z_{i}$. Overall, the qualitative behavior of the LNL fluxes obtained based on the more subjective Fourier method is the same as that found previously with the SST07 method, as well as from two other more sophisticated CS methods presented in appendix B.

\section{Discussion}

Comparing the GCG parameterization in section $3 \mathrm{a}$ and the LNL decomposition in section $3 b$, it is clear that in the CBL, the gradient diffusion flux is not a good representation of the local flux in terms of physics, nor is the countergradient correction flux of the nonlocal flux. The mismatch is especially prevalent in the upper mixed layer between $0.4 z_{i}$ and $0.8 z_{i}$, where the gradient flux and the local flux are in opposite directions (i.e., contradicting signs), and the countergradient flux exceeds the net flux while the nonlocal flux remains less than the net flux. Although only two PBL schemes are tested, the aforementioned mismatch is expected for PBL schemes under the GCG framework in general. This is because the downward gradient flux above $0.4 z_{i}$ is a direct consequence of $\partial\langle\theta\rangle / \partial z$ switching from negative below to positive above (see Fig. 2a), irrespective of the particular choice of eddy diffusivity $K_{H}$, so long as it is positive. In other words, if a countergradient correction-based PBL scheme achieves first-order agreement with the benchmark LES with respect to the vertical profile of $\bar{\theta}(z)$ (note that this is to be expected for those PBL schemes, since reproducing the countergradient behavior $\partial \bar{\theta} / \partial z \times \overline{w^{\prime} \theta^{\prime}}>0$ is exactly what they are designed for), its gradient flux $-K_{H} \partial \bar{\theta} / \partial z$ must be negative between $0.4 z_{i}$ and $0.8 z_{i}$. Meanwhile, the larger-than-net countergradient flux $K_{H} \gamma$ must also be present to compensate for the negative gradient flux.

At a more fundamental level, if turbulence is viewed as the choice of nature for mixing, high efficiency must be expected for turbulent processes. Since eddy structures in the CBL are classically viewed as consisting of organized nonlocal thermals and smaller local eddies (see Fig. 1), a heat flux efficiency (HFE) parameter can be defined to measure the coordination of large and small turbulent eddies for mixing heat in the following manner:

$$
\begin{aligned}
\operatorname{HFE}(z) & \equiv \frac{\mid \overline{w^{\prime} \theta^{\prime}}}{\mid \overline{w^{\prime} \theta^{\prime}}}(z)+\overline{w^{\prime} \theta^{\prime}}(z)|+| \overline{w^{\prime} \theta^{\prime}}(z) \mid \\
& =\frac{\left|\overline{w^{\prime} \theta^{\prime}}(z)\right|}{\left|\overline{w^{\prime} \theta_{\mathrm{NL}}^{\prime}}(z)\right|+\left|\overline{w^{\prime} \theta^{\prime}}(z)\right|},
\end{aligned}
$$

where subscripts NL and L represents nonlocal and local fluxes separated by a certain CS method. From the perspective of the Fourier decomposition, where heat fluxes are associated with a spectrum of wavenumbers, the corresponding HFE can be defined as

$\operatorname{HFE}^{\text {Fourier }}(z)=\frac{\left|\int_{0}^{k_{g}} F_{w \theta}(k, z) d k\right|}{\int_{0}^{k_{g}}\left|F_{w \theta}(k, z)\right| d k}=\frac{\left|\overline{w^{\prime} \theta^{\prime}}(z)\right|}{\int_{0}^{k_{g}}\left|F_{w \theta}(k, z)\right| d k}$,

where $k_{g}$ is the grid cutoff wavenumber representing $2 \Delta_{h}$ waves and $\Delta_{h}$ is the horizontal grid spacing. Note that 

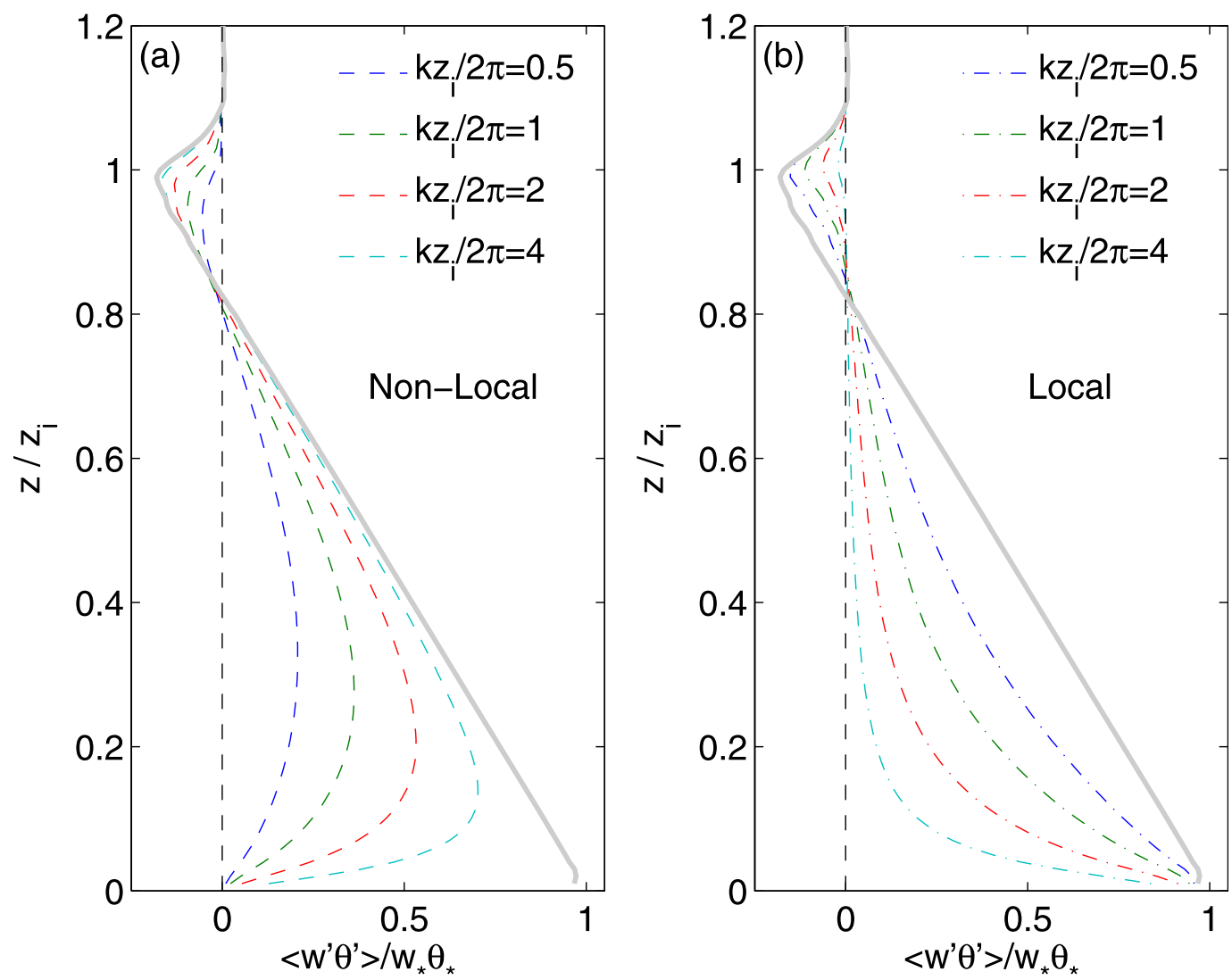

FIG. 8. Vertical profiles of the time- and case-averaged (a) nonlocal and (b) local heat fluxes from Fourier decomposition for $k z_{i} / 2 \pi=0.5,1,2$, and 4 .

the absolute sign in the denominator is placed around $F_{w \theta}$ inside the integral, meaning that the magnitudes of all cospectral density are integrated. Efficient mixing processes are expected to have an HFE close to unity. That is to say, to achieve an overall upward heat flux, the most efficient mixing processes must have eddies of all sizes contributing positively in a concerted fashion, which gives an HFE of 1 . Less efficient mixing with a smaller HFE means that at least some eddies are transporting heat in the opposite direction of the net heat flux. In this case, the rest of the eddies would have to transport an extra amount of heat upward to make up for the negative contributions, if the overall heat flux stays the same.

Figure 9 presents the vertical profiles of HFE computed based on Eqs. (7) and (8). The SST07 method with $p=10 \%$ is adopted to separate LNL fluxes for Eq. (7). The results are time and case averaged. In Fig. 9, both HFEs are identically unity below $\sim 0.8 z$, indicating the most coordinated and efficient turbulent processes among eddies of all sizes to achieve upward mixing of heat. Approaching $0.8 z_{i}$ where the net heat flux is close to zero (see Fig. 2b), HFE is not well defined since both the numerators and denominators of Eqs. (7) and (8) go to zero. At around $1.0 z_{i}$, both HFEs diagnosed from LES recover toward unity before falling again to around 0.5 at $1.1 z_{i}$. The reason for the decrease of efficiency above $1.0 z_{i}$ is not clear. However, note that the net heat fluxes decrease rapidly above $1.0 z_{i}$ and diminish slightly below $1.1 z_{i}$ for all cases (see Fig. 2b). Therefore, the HFEs above $1.1 z_{i}$ are associated with large uncertainties. Other $p$ values in the range of $1 \%-50 \%$ are also tested, and give essentially identical results (i.e., HFE $=1$ ) within most part of the CBL (not shown). The insensitivity of HFE to the free parameter $p$ suggests the robustness of the results.

In terms of PBL schemes, a similar parameter can be defined for countergradient correction-based schemes, where

$$
\operatorname{HFE}^{\mathrm{PBL}}(z) \equiv \frac{\left|\overline{w^{\prime} \theta_{g}^{\prime}}(z)+\overline{w^{\prime} \theta_{\mathrm{cg}}^{\prime}}(z)\right|}{\left|\overline{w^{\prime} \theta_{g}^{\prime}}(z)\right|+\left|\overline{w^{\prime} \theta_{\mathrm{cg}}^{\prime}}(z)\right|} .
$$

For the YSU scheme, both the numerator and the denominator of Eq. (9) include an additional entrainment term $\overline{w^{\prime} \theta^{\prime}}$ ent $(z)$ and becomes 


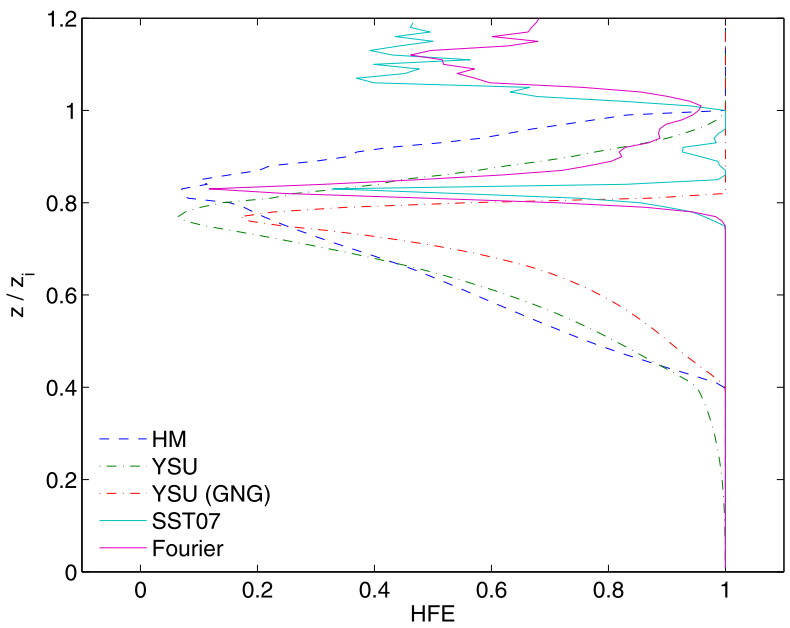

FIG. 9. Vertical profiles of time- and case-averaged heat flux efficiency for LES diagnosed LNL fluxes and the fluxes from PBL schemes. For the YSU(GNG) case, the countergradient and entrainment fluxes are grouped together as a nongradient term. The HFE based on the SST07 method uses $p=10 \%$.

$\operatorname{HFE}^{\mathrm{YSU}}(z) \equiv \frac{\left|\overline{w^{\prime} \theta_{g}^{\prime}}(z)+\overline{w^{\prime} \theta_{\mathrm{cg}}^{\prime}}(z)+\overline{w^{\prime} \theta_{\mathrm{ent}}^{\prime}}(z)\right|}{\left|\overline{w^{\prime} \theta_{g}^{\prime}}(z)\right|+\left|\overline{w^{\prime} \theta_{\mathrm{cg}}^{\prime}}(z)\right|+\left|\overline{w^{\prime} \theta_{\mathrm{ent}}^{\prime}}(z)\right|}$.

The time- and case-averaged HFE parameters for the two PBL schemes are also presented in Fig. 9. For the $\mathrm{HM}$ formulation, $\mathrm{HFE}^{\mathrm{HM}}$ drops at around $0.4 z_{i}$, where the gradient flux turns negative and the countergradient flux exceeds the total flux. HFE decreases to zero at $0.8 z_{i}$, and slowly recovers to unity at the CBL top. Above $1.0 z_{i}, \mathrm{HFE}^{\mathrm{PBL}}=1$ by definition, since the countergradient correction term is not defined above $1.0 z_{i}$, leaving the gradient-diffusion term solely responsible for the heat fluxes. In comparison, $\mathrm{HFE}^{\mathrm{YSU}}$ drops at an even lower elevation, in fact right above the surface, due to the presence of the negative entrainment flux. Between $\sim 0.4 z_{i}$ and $\sim 1.0 z_{i}, \mathrm{HFE}^{\mathrm{YSU}}$ is close to $\mathrm{HFE}^{\mathrm{HM}}$, except that minimum efficiency is reached a lower elevation slightly below $\sim 0.8 z_{i}$.

In the YSU scheme, both the countergradient and entrainment terms are there to correct for nonlocal mixing effects (Hong et al. 2006). Therefore they can be grouped together and treated as a nongradient term:

$\mathrm{HFE}^{\mathrm{YSU}(\mathrm{GNG})}(z) \equiv \frac{\left|\overline{w^{\prime} \theta_{g}^{\prime}}(z)+\left[\overline{w^{\prime} \theta_{\mathrm{cg}}^{\prime}}(z)+\overline{w^{\prime} \theta^{\prime}}{ }_{\mathrm{ent}}(z)\right]\right|}{\left|\overline{w^{\prime} \theta_{g}^{\prime}}(z)\right|+\left|\overline{w^{\prime} \theta_{\mathrm{cg}}^{\prime}}(z)+\overline{w^{\prime} \theta^{\prime}}{ }_{\mathrm{ent}}(z)\right|}$.

HFE for the YSU scheme recomputed based on gradient and nongradient (GNG) partitions is also presented in Fig. 9. The resulting HFE profile is closer to the LES benchmark. It drops at the same elevation as $\mathrm{HFE}^{\mathrm{HM}}$ at $\sim 0.4 z_{i}$. In the upper mixed layer, although slightly larger than $\mathrm{HFE}^{\mathrm{HM}}$ and $\mathrm{HFE}^{\mathrm{YSU}}$, $\mathrm{HFE}^{\mathrm{YSU}(\mathrm{GNG})}$ drops again toward zero approaching $0.8 z_{i}$. Higher up, $\mathrm{HFE}^{\mathrm{YSU}(\mathrm{GNG})}$ increases rapidly to 1 . The large efficiency for the GNG partition of the YSU scheme above $0.8 z_{i}$ is mainly due to the dominance of the entrainment term over the countergradient term, making the overall nongradient contribution negative, which is the same sign as the gradient term there (see Fig. 3b). However, in the HM scheme, the entrainment flux is contributed by the both gradient and countergradient terms with opposite signs (Fig. 3a), leading to smaller HFEs. When $\mathrm{HFE}^{\mathrm{YSU}(\mathrm{GNG})}$ is compared to HFE ${ }^{\mathrm{YSU}}$, it is apparent that the GNG fluxes are a conceptual improvement over the GCG fluxes. However, the main issue of reduced efficiency in the upper mixed layer still exists for the GNG fluxes. Another issue with the entrainment term raised earlier in section 3 is that the parameterized entrainment flux in Fig. 3a and LES diagnosed nonlocal environment flux in Fig. $5 \mathrm{c}$ are in opposite directions. This suggest some conceptual misrepresentation of the entrainment processes.

Overall, the parameterized GCG processes, regardless of their physical representations, are less efficient in the upper mixed layer and lower entrainment zone. The reduced efficiency is a result of GCG fluxes counteracting each other, whereas CBL eddies are much more coordinated. This suggests a conceptual mismatch between the parameterized and the actual turbulent mixing processes in the CBL. Therefore, the countergradient correction term in Eq. (2) should be best viewed as a technical fix to an already problematic eddy-diffusion concept [Eq. (1)] in the representation of CBL turbulence.

For PBL schemes relying on the GCG framework, results presented here suggest that although they may be able to produce the correct net heat flux profiles (see section 3a), they are conceptually problematic in terms of their misrepresentation of turbulent mixing. It is the issue of whether the end justifies the means that we wish to point out through this study. We argue that the end results, in this case the correct potential temperature and heat flux profiles, should not be the only aim of a PBL scheme. Meaningful physical representation of the CBL mixing processes is equally important, if not more so. By taking account of the actual effects of LNL eddies, we might be able to construct a physically insightful PBL scheme that also has good practical performance. Here we note the scale-aware PBL scheme of Shin and Hong (2015), which took a different approach to account for nonlocal mixing. They adopted a nonlocal flux profile (see their Fig. 2) obtained from conditional sampling of LES to parameterize nonlocal fluxes. Similar to Fig. 5, the magnitude of their nonlocal flux profile obtained from CS is smaller than the net flux at all elevations. As a result, 
their parameterized local and nonlocal fluxes are both positive below $\sim 0.8 z_{i}$ (see their Figs. $5 \mathrm{c}$ and $5 \mathrm{~d}$ ) and negative above, suggesting much improved heat flux efficiency. However, although it is not clear from their results, this is most likely associated with a cost of the loss of positive potential temperature gradient in the upper mixed layer, since the gradient-diffusion term is still used for parameterizing local fluxes in their new scheme.

\section{Summary and future work}

In the class of countergradient correction-based PBL schemes outlined by Eq. (2), a countergradient term $K_{H} \gamma_{\mathrm{cg}}$ is added alongside the gradient diffusion term $-K_{H} \partial \bar{\theta} / \partial z$ to correct for nonlocal mixing in the $\mathrm{CBL}$, so that the desired counterpotential temperature gradient heat flux profiles can be reproduced. This study examines the representation of nonlocal thermals by $K_{H} \gamma_{\mathrm{cg}}$ and local eddies by $-K_{H} \partial \bar{\theta} / \partial z$, not based on the a posteriori performance of PBL schemes but rather in terms of physical representations. The evaluation is based on fine-resolution wide-domain LESs of six idealized CBL cases with different amounts of surface heating, geostrophic wind speeds, and shear. The simulated CBLs develop some kind of organized nonlocal convective structures in the form of cells and horizontal roll vortices depending on the relative strength of heating to shear.

Using statistics obtained from horizontal averaging of LES data, the GCG heat flux profiles of two popular PBL schemes are diagnosed a priori. It is shown that in the upper mixed layer and the entrainment zone, the gradient and countergradient heat fluxes are of opposite signs. The gradient flux is negative, indicating downward heat transport as a result of the positive background potential temperature gradient above $\sim 0.4 z_{i}$. In response, the countergradient term exceeds the net upward heat flux between $0.4 z_{i}$ and $0.8 z_{i}$ to compensate for the negative contribution by the gradient flux. These conclusions are not limited to the two particular schemes and should apply to GCG-based PBL schemes in general.

The LNL fluxes are diagnosed by conditional sampling of the LES flow field. In contrast to the GCG fluxes, the LNL fluxes are in the same directions within most parts of the CBL (i.e., both fluxes are positive below $\sim 0.8 z_{i}$ and negative above). To further illustrate the weakness of the GCG representation to turbulent fluxes in the CBL, the heat flux cospectra are computed in search for eddies that contribute negatively toward an overall positive heat flux. However, it is found that except for a narrow region around $0.8 z_{i}$ where positive (at large wavenumbers) and negative (at small wavenumbers) values coexist, the cospectral density for eddies of all sizes share the same sign as the total flux at that vertical level. In particular, small eddies between $0.4 z_{i}$ and $0.8 z_{i}$ carry upward heat fluxes against a positive background $\partial\langle\theta\rangle / \partial z$. In appendix B, two sophisticated CS methods are conducted for the BT case. Both yield LNL profiles that are qualitatively similar to those presented in section $3 \mathrm{~b}$.

Having investigated the physical insights for Eq. (2)type PBL schemes, many questions still remain to be examined in the future. For example, why do small eddies cause upward heat flux when the overall $\partial \bar{\theta} / \partial z$ is positive? If the gradient formulation is a poor representation of the local flux, then what is a more physically meaningful parameterization? To answer these questions, one needs to closely examine the eddy structures of the CBL. One alternative hypothesis is that local mixing could be associated with the vertical gradient of the updraft potential temperature $\overline{\theta_{u}}(z)$ rather than $\bar{\theta}(z)$. Judging from Fig. $4 \mathrm{a}, \partial \overline{\partial u} / \partial z$ switches sign at about $0.8 z_{i}$ for updraft identified by the $p=10 \%$ criterion. Gradient flux computed this way is at least positive, which ensures high efficiency. The physical reasoning behind this could be that it is the small eddies within the updraft, rather than the environment, that are responsible for the most of the local fluxes. However, a close examination of subupdraft and subenvironmental local fluxes in Fig. $6 \mathrm{~d}$ seems to contradict this view. The updrafts associated local fluxes contribute very little compared to local fluxes in the environment, at least for $p=10 \%$.

Acknowledgments. We thank Prof. Ming Xue and Dr. Jian-feng Gu for helpful discussions. We are grateful for Dr. Stefano Serafin for sharing his literature review on the GCG formulations. We thank the High Performance Computing Center (HPCC) of Nanjing University for the IBM Blade cluster system, where numerical calculations were performed. This work was supported by the National Key R\&D Program of China under Grant 2017YFC1501601. Bowen Zhou, Shiwei Sun, and Kai Yao were supported by the National Natural Science Foundation of China (Grant 41505047) and a collaborative project on PBL parameterizations with the Nanjing Center for Atmospheric Research (NJCAR). Kefeng Zhu was supported by the Foundation of China Meteorological Administration (Grant GYHY201506006).

\section{APPENDIX A}

\section{Formulations of the PBL Schemes}

In the following, formulations of the HM and YSU schemes for the CBL are briefly summarized. Variables already explained in the main text are not repeated here for brevity. In Holtslag and Moeng (1991), the countergradient term $\gamma_{\mathrm{cg}}$ and the eddy diffusivity $K_{H}$ are 


$$
\begin{aligned}
\gamma_{\mathrm{cg}} & =\frac{g}{\theta_{0}} \frac{\overline{\theta^{\prime 2}}}{\overline{w^{\prime 2}}}, \\
K_{H} & =w_{*} z_{i}\left(\frac{z}{z_{i}}\right)^{4 / 3}\left(1-\frac{z}{z_{i}}\right)^{2}\left(1+R_{H} \frac{z}{z_{i}}\right),
\end{aligned}
$$

where $\overline{\theta^{\prime 2}}$ and $\overline{w^{\prime 2}}$ are the variances of the perturbation potential temperature $\theta^{\prime}$ and vertical velocity $w^{\prime}$, whose vertical profiles are fitted from observations (Lenschow et al. 1980); $g$ is the gravitational constant, $\theta_{0}$ is a reference potential temperature; and $R_{H}(=-0.2)$ is a prescribed ratio of the entrainment flux to the surface flux for heat.

The YSU scheme (Hong et al. 2006) includes an entrainment term in addition to the GCG fluxes:

$$
\overline{w^{\prime} \theta^{\prime}}=-K_{H}\left(\frac{\partial \bar{\theta}}{\partial z}-\gamma_{\mathrm{cg}}\right)+\overline{w^{\prime} \theta^{\prime}}\left(\frac{z}{z_{i}}\right)^{3},
$$

where $\overline{w^{\prime} \theta^{\prime}}$ is the entrainment flux at the top of the CBL (i.e., $1.0 z_{i}$ ). The terms $K_{H}$ and $\gamma_{\text {cg }}$ are formulated as

$$
\begin{aligned}
\gamma_{\mathrm{cg}} & =6.8 \frac{\overline{w^{\prime} \theta_{s}^{\prime}}}{w_{s 0} z_{i}}, \\
K_{H} & =\kappa w_{s} z\left(1-\frac{z}{z_{i}}\right)^{2} \operatorname{Pr},
\end{aligned}
$$

where $\overline{w^{\prime} \theta^{\prime}}$ is the surface heat flux, $w_{s 0}$ is the mixed-layer velocity scale $w_{s}$ evaluated at $0.5 z_{i}$. The vertical profile of $w_{s}$ is defined as

$$
w_{s}=\left(u_{*}^{3}+\phi_{m} \kappa w_{*} z / z_{i}\right)^{1 / 3},
$$

where $u_{*}$ is the friction velocity and $\kappa(=0.4)$ is the von Kármán constant. Also, $\phi_{m}$ is the dimensionless shear evaluated at the top of the surface layer at $0.1 z_{i}$ :

$$
\phi_{m}=\frac{\kappa z}{u_{*}} \frac{d \bar{U}}{d z}=\left(1-16 \frac{0.1 z_{i}}{L}\right)^{-1 / 4}
$$

where $U$ is the wind speed. In Eq. (A5), Pr is the turbulent Prandtl number

$$
\operatorname{Pr}=1+\left(\operatorname{Pr}_{0}-1\right) \exp \left[-3\left(\frac{z}{z_{i}}-0.1\right)^{2}\right],
$$

and $\operatorname{Pr}_{0}=1.272$ is the surface Pr. In Eq. (A3), the entrainment flux $\overline{w^{\prime} \theta^{\prime}} z_{i}$ is formulated as

$$
\overline{w^{\prime} \theta^{\prime}}{ }_{z_{i}}=-e_{1} w_{m}^{3} / z_{i}
$$

where $e_{1}=4.5 \mathrm{~m}^{-1} \mathrm{~s}^{-2} \mathrm{~K}^{-1}$ is a dimensional coefficient, and $w_{m}=\left(w_{*}^{3}+5 u_{*}^{3}\right)^{1 / 3}$ is a velocity scale based on
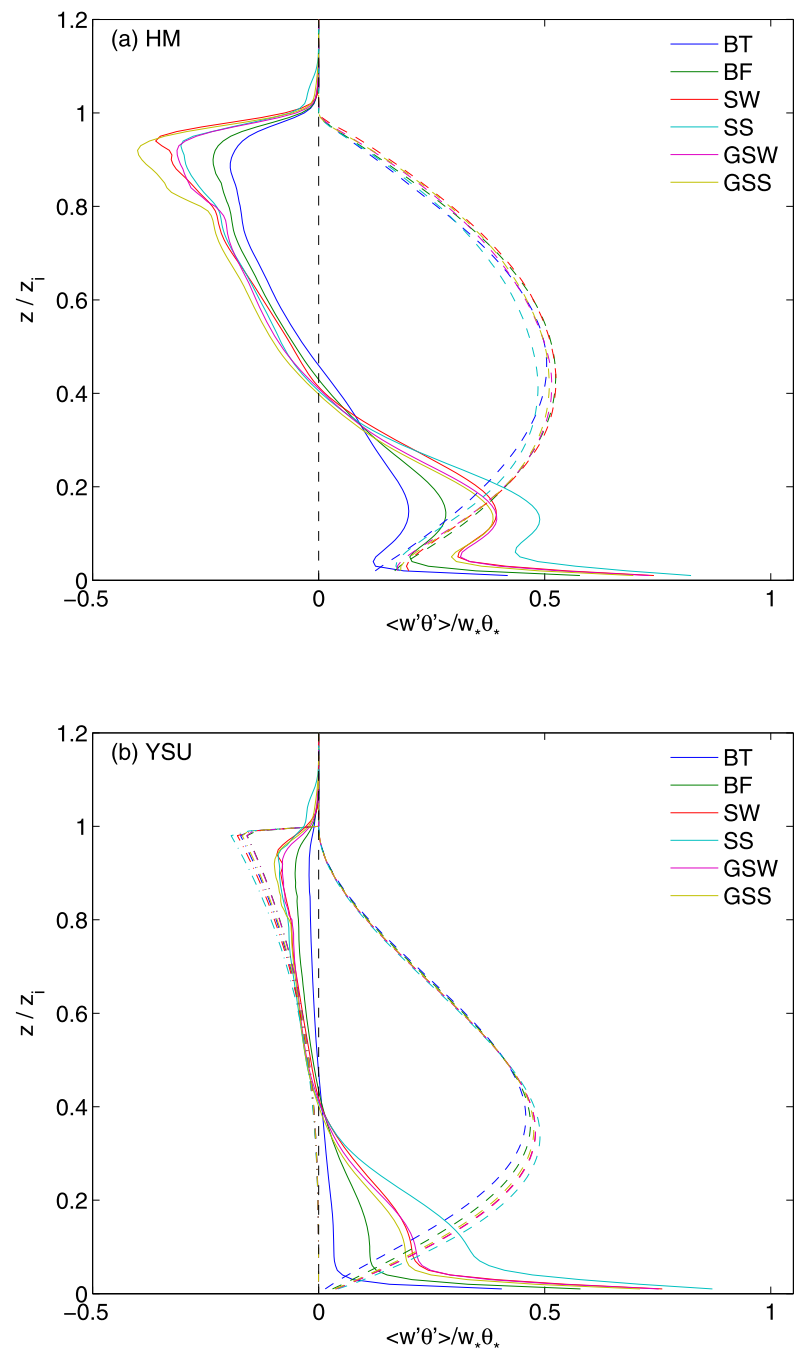

FIG. A1. As in Fig. 3, but for all six cases. Solid lines represent directly computed gradient fluxes, dashed lines represent countergradient fluxes, and dash-dotted lines in (b) represent entrainment fluxes for the YSU scheme.

surface-layer turbulence. The boundary layer depth $z_{i}$ in both the HM and YSU schemes are set to the LES diagnosed values for consistency.

The directly computed gradient fluxes for the HM and YSU schemes for all six cases are presented in Fig. A1.

\section{APPENDIX B}

\section{Diagnosing LNL Fluxes with Tracers}

Using vertical velocity alone to identify coherent structures in the CBL according to Eq. (4) assumes a constant fractional area covered by thermals. This is not realistic because as thermals rise within the CBL, the fractional area should decrease with height due to 


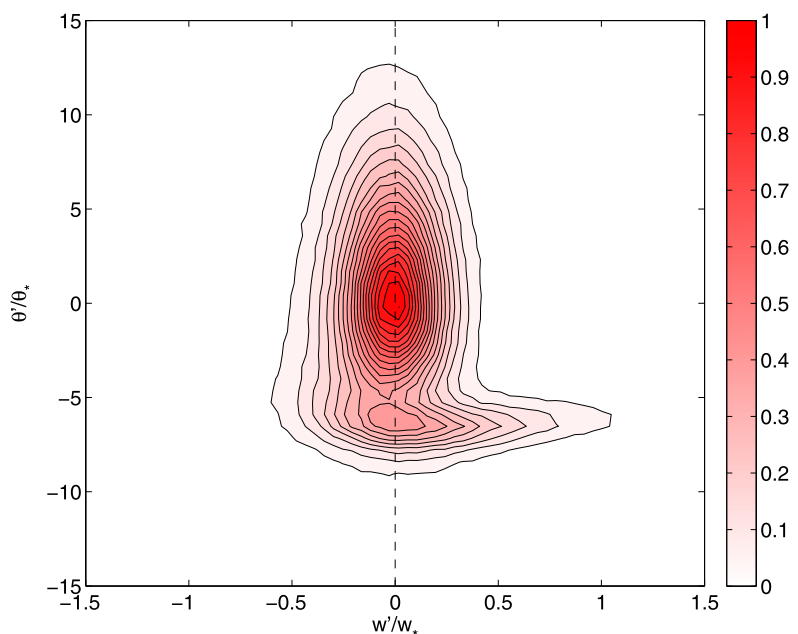

FIG. B1.2D histogram of normalized $w^{\prime}$ and $\theta^{\prime}$ at $1.0 z_{i}$. The contour level is 0.1 .

turbulent entrainment of surrounding air and subsequent loss of active buoyancy (SST07; Romps and Charn 2015). A better way to identify thermals in CBLs simulated by LES is to introduce numerical tracers into the flow field (Couvreux et al. 2010; Shin and Hong 2013; Park et al. 2016). In this appendix, we adopt two of the abovementioned conditional sampling methods to separate between local and nonlocal eddies. Given the overlapping flux profiles among cases based on the SST07 method and due to computational cost constraints, these two tracerbased methods are performed for the BT case only.
In the LES domain, a conservative tracer is emitted at the first grid point above the surface by setting its concentration to unity. A decaying tracer of Couvreux et al. (2010) is also injected, with a half-life of $3.33 \tau$, where $\tau$ is the CBL turnover time introduced in section 2 . The results are qualitatively similar to that of the conservative tracer, hence not shown. This is mainly because of the short integration time of $4 t_{0}\left(t_{0}=6 \tau\right)$, such that oversaturation of the passive tracer has not yet occurred by the end of the simulation. In the following, all vertical profiles presented are horizontally averaged and time averaged.

The first conditional sampling criteria for thermals are based on Couvreux et al. (2010), but more stringent:

$$
\begin{aligned}
w^{\prime}(x, y, z) & >\sigma_{w}(z) \quad \text { and } \\
c^{\prime}(x, y, z) & >\max \left[\sigma_{c}(z), c_{p}(z)-\langle c(z)\rangle\right],
\end{aligned}
$$

where $c$ is the tracer concentration, $c_{p}(z)$ is the higher peak concentration of $c$ (which will be explained shortly), and primes represent perturbations from the horizontal mean. The choice of $w^{\prime}>\sigma_{w}$ over $w^{\prime}>0$ as in the original Couvreux et al. (2010) study is to identify the updraft cores and to filter out incoherent eddies with small $w^{\prime}$ (Park et al. 2016). Additionally, $w^{\prime}>\sigma_{w}$ is advantageous in the entrainment zone where gravity waves can be mistaken as updrafts. As shown in Fig. B1, the joint distribution of $w^{\prime}$ and $\theta^{\prime}$ at $1.0 z_{i}$ is of a bimodal structure. The cold-temperature mode (i.e., $\theta^{\prime} / \theta_{*}<-5$ ) corresponds to the updrafts with $w^{\prime}>0$, and the subsiding

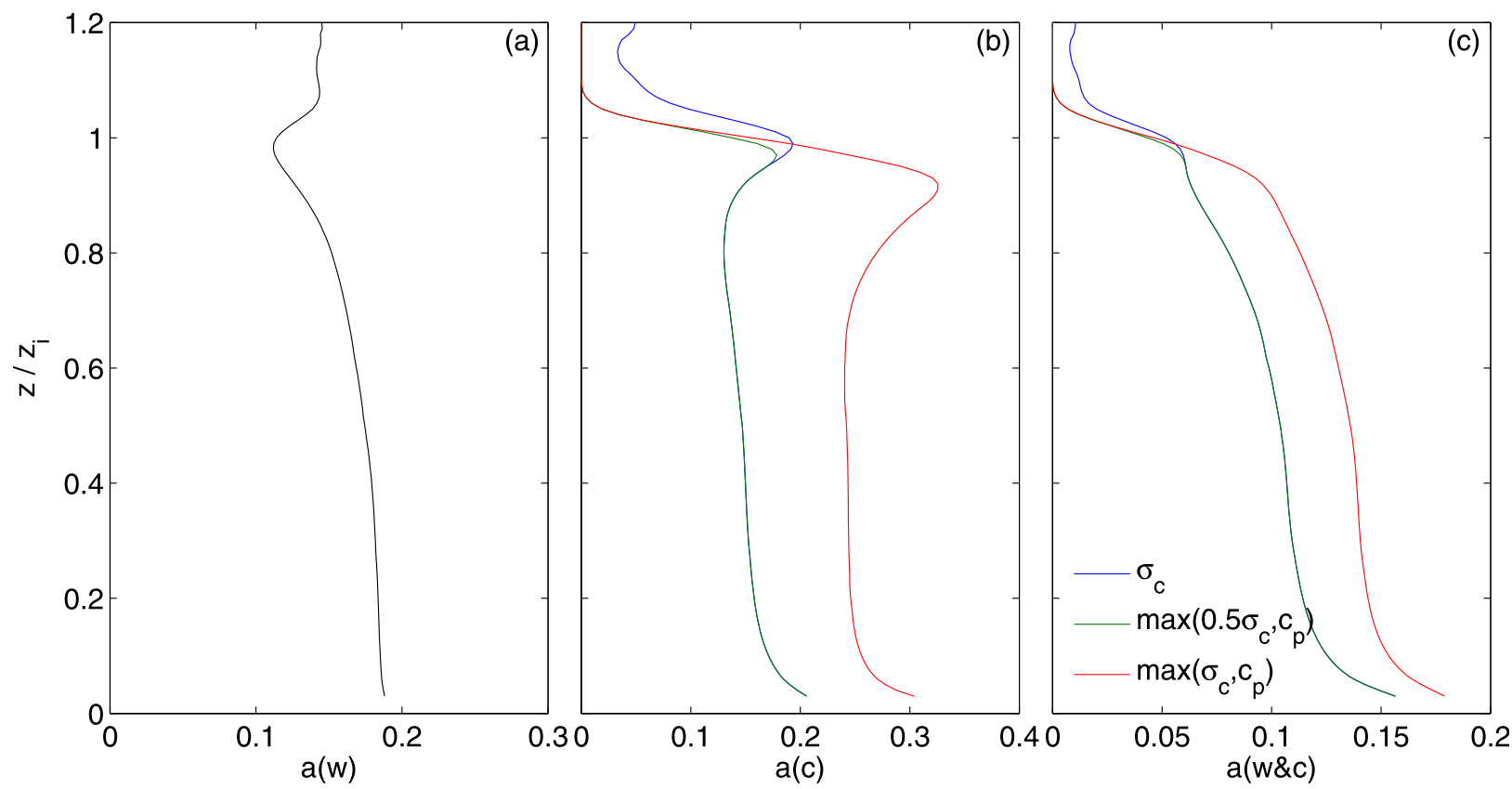

FIG. B2. Fractional area of updrafts identified with (a) $w$, (b) $c$, and (c) $w$ and $c$ together. 


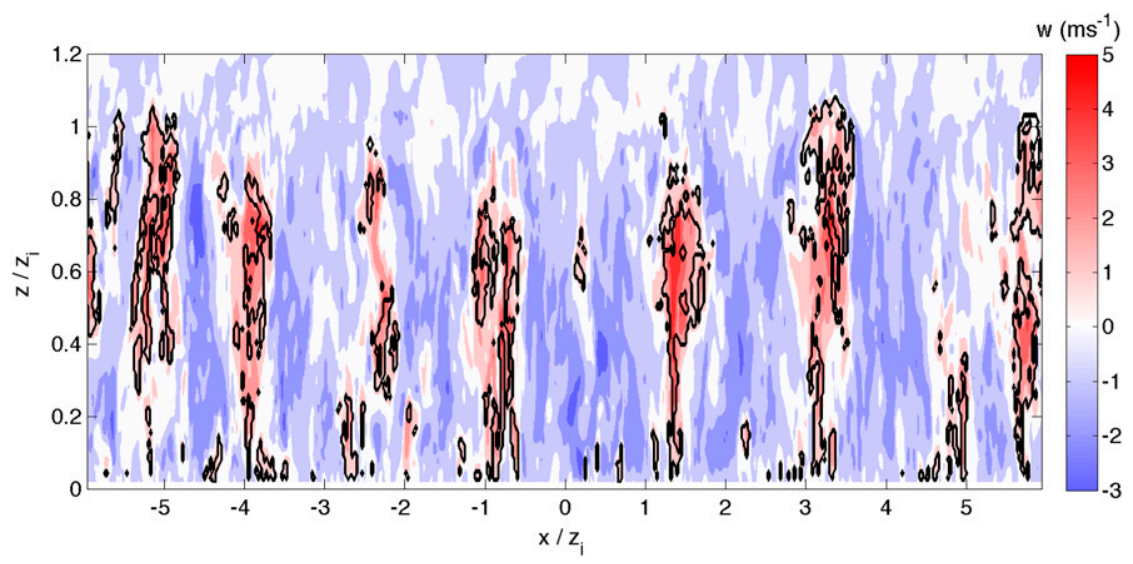

FIG. B3. Contours of $w$ along a $y-z$ vertical cross section at $7200 \mathrm{~s}$. The contour interval is $1 \mathrm{~m} \mathrm{~s}^{-1}$. The black contour lines enclose thermals identified with Eq. (B1).

shells with $w^{\prime}<0$. The higher temperature mode is nearly symmetrical around $w^{\prime}=0$. It mainly consists of small-scale oscillations (mostly waves) under the stably stratified background. Above $1.0 z_{i}$, the lowertemperature mode quickly diminishes, making it even harder to separate thermals from waves. Setting $w^{\prime}>0$ as one of the conditional sampling criteria would overestimate updrafts, hence the use of $w^{\prime}>\sigma_{w}$. However, $w^{\prime}>\sigma_{w}$ is still insufficient to adequately identify thermals. As shown in Fig. B2a, the thermal fraction $a(w)$ still increases with height above $1.0 z_{i}$, although much less rapidly than the $w^{\prime}>0$ criterion (results not shown). Such increase is unphysical since the thermal fraction should decrease monotonically with height in the presence of entrainment in a dry CBL.

For the $c^{\prime}$ criterion in Eq. (B1), another parameter $c_{p}$ is introduced for the purpose of improving conditional sampling in the entrainment zone. The value of $c_{p}(z)$ is computed by first identifying the peak(s) of the tracer distribution at height $z$. Below $\sim 0.8 z_{i}$, the tracer distribution is generally single-peaked, so that $c_{p}(z)$ is simply the mode of $c$. In the entrainment zone, the distribution
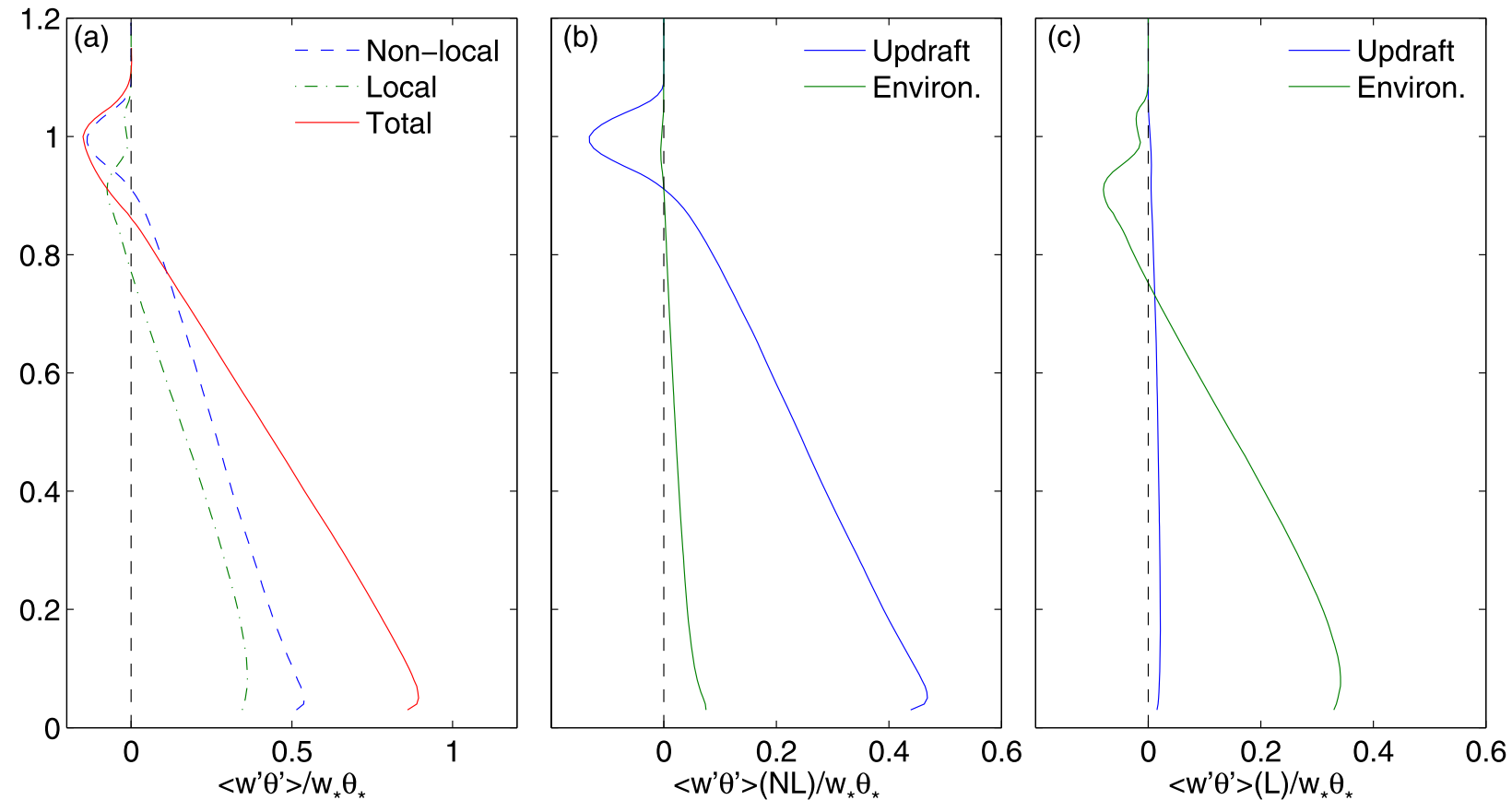

FIG. B4. Vertical profiles of normalized (a) local and nonlocal contributions to the total flux. (b),(c) The updraft and environmental contributions to the nonlocal and local fluxes, respectively. 
TABLE B1. Definition of quadrants based on $w^{\prime}$ and $c^{\prime}$, where $c^{\prime}=c-\left[\langle c\rangle+\max \left(\sigma_{c}, c_{p}\right)\right]$.

\begin{tabular}{ccccc}
\hline \hline & Updraft & Ascendance & Downdraft/shell & Subsidence \\
\hline$w^{\prime}$ & + & + & - & - \\
$c^{\prime}$ & + & - & + & - \\
\hline
\end{tabular}

of $c$ becomes bimodal, with one peak at a lower concentration representing tracers that have been diffused upward. The second peak is at a higher concentration representing tracers that have been advected by thermals. Then $c_{p}$ takes on the value of $c$ at the higher peak associated with thermals. By setting $c^{\prime}>\sigma_{c}$ alone, the thermal area $a(c)$ is overestimated above $\sim 0.9 z_{i}$ as shown by the blue curve in Fig. B2b (see also Shin and Hong 2013, their Fig. 7a). By accounting for $c_{p}$, the increase of $a(c)$ with height above $\sim 0.9 z_{i}$ is reduced, and $a(c)$ quickly drops to zero at $\sim 1.1 z_{i}$ in accordance with the highest elevation reached by overshooting thermals (Garcia and Mellado 2014).

The fractional area of thermals computed according to Eq. (B1) is presented in Fig. B2c. The additional $c_{p}$ criterion mainly improves the profile above $\sim 0.9 z_{i}$. The overall thermal fraction decreases from $15 \%$ in surface layer to about $5 \%$ near the top of the CBL. To force monotonic decrease of the thermal fraction with height, another criterion can be implemented where $\sigma_{c}$ in Eq. (B1) is replaced with $0.5 \sigma_{c}$. Although in this case $a(c)$ increases with height between $0.1 z_{i}$ and $0.8 z_{i}$ as shown in Fig. B2b, the thermal fraction $a(c \& w)$ strictly decreases upward in Fig. B2c. To visualize the conditionally sampled thermals, a vertical cross section of $w$ is presented in Fig. B3. Thermals enclosed by the black contours mostly overlap with strong updrafts.

The LNL flux decomposition based on Eq. (B1) is presented in Fig. B4a. Consistent with the data in Fig. 5, both LNL fluxes are positive below $\sim 0.8 z_{i}$, and negative above $\sim 0.9 z_{i}$. In the small section in between where the magnitude of the total flux is close to zero, the LNL fluxes are of opposite signs. In the mixed layer, the nonlocal flux is slightly larger than the local flux, while in the entrainment zone the nonlocal flux dominates. Further partition of the LNL fluxes into updraft and environment contributions reveals a qualitatively similar picture as Figs. $6 \mathrm{c}$ and $6 \mathrm{~d}$. The updraft contribution is mostly responsible for the nonlocal flux as shown in
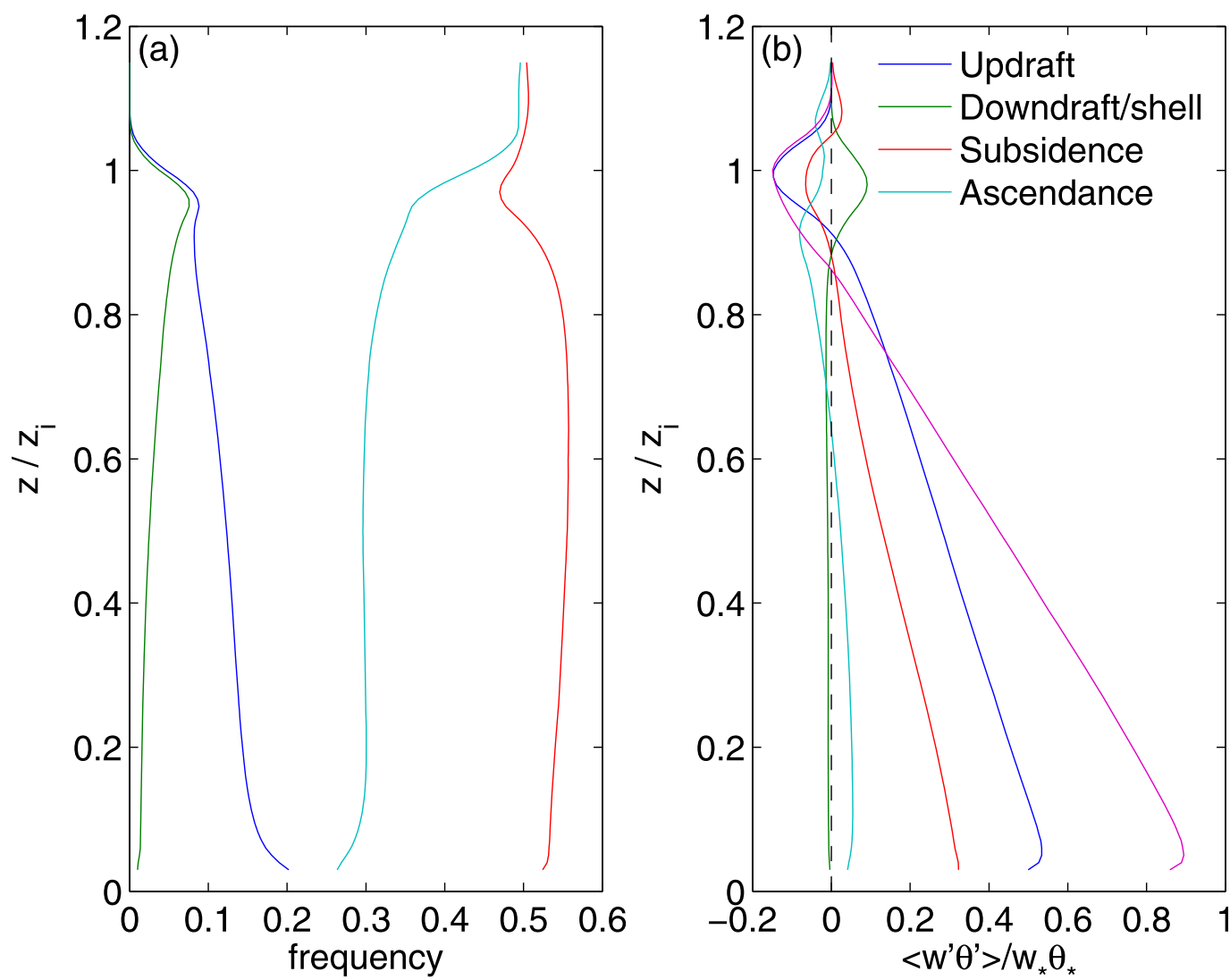

FIG. B5. Vertical profiles of (a) frequency and (b) normalized heat fluxes of updrafts, downdrafts, subsidence, and ascendance. 
Fig. B4b, whereas the subenvironmental variability is the dominant component of the local flux (Fig. B4c).

Recently, Park et al. (2016) used two decaying tracers and the vertical velocity to separate flow structures into octants. Here, we adopt a simplified version of their method based on one tracer to further separate ascendance from updrafts, and subsiding shells from environmental subsidence. The conditional sampling criteria are presented in Table B1. The definition of $c^{\prime}$ is modified from Park et al. (2016) to capture nonlocal advective updrafts, especially above the mixed layer. As shown in Fig. B5a, the overall frequencies of ascendance and subsidence are most prevalent in the entrainment zone corresponding to the presence of gravity waves. The coverage area of downdraft or subsiding shells are smallest throughout the CBL in Fig. B5a, although its contribution to fluxes are significant in the entrainment zone in Fig. B5b. This is expected because as thermals encroach on the stably stratified layer, the downdraft/ shell represents the returning part of a vortex-like structure, with high $c$ and negative $w$ (Park et al. 2016). Such quadrant sampling reveals more information on the coherent structures of the CBL. The overall conclusions of this study still hold according to Fig. B5b.

\section{REFERENCES}

Bougeault, P., and P. Lacarrere, 1989: Parameterization of orography-induced turbulence in a mesobeta-scale model. Mon. Wea. Rev., 117, 1872-1890, https://doi.org/10.1175/15200493(1989)117<1872:POOITI>2.0.CO;2.

Brown, A. R., and A. L. M. Grant, 1997: Non-local mixing of momentum in the convective boundary layer. Bound.-Layer Meteor., 84, 1-22, https://doi.org/10.1023/A:1000388830859.

Chow, F. K., R. L. Street, M. Xue, and J. H. Ferziger, 2005: Explicit filtering and reconstruction turbulence modeling for largeeddy simulation of neutral boundary layer flow. J. Atmos. Sci., 62, 2058-2077, https://doi.org/10.1175/JAS3456.1.

Couvreux, F., F. Hourdin, and C. Rio, 2010: Resolved versus parametrized boundary-layer plumes. Part I: A parametrizationoriented conditional sampling in large-eddy simulations. Bound.-Layer Meteor., 134, 441-458, https://doi.org/10.1007/ s10546-009-9456-5.

Cuijpers, J. W. M., and A. A. M. Holtslag, 1998: Impact of skewness and nonlocal effects on scalar and buoyancy fluxes in convective boundary layers. J. Atmos. Sci., 55, 151-162, https://doi.org/ 10.1175/1520-0469(1998)055<0151:IOSANE > 2.0.CO;2.

Deardorff, J. W., 1966: The counter-gradient heat flux in lower atmosphere and in laboratory. J. Atmos. Sci., 23, 503-506, https:// doi.org/10.1175/1520-0469(1966)023<0503:TCGHFI>2.0.CO;2.

_ 1972: Theoretical expression for the countergradient vertical heat flux. J. Geophys. Res., 77, 5900-5904, https://doi.org/ 10.1029/JC077i030p05900.

Ebert, E. E., U. Schumann, and R. B. Stull, 1989: Nonlocal turbulent mixing in the convective boundary layer evaluated from large-eddy simulation. J. Atmos. Sci., 46, 2178-2207, https://doi.org/10.1175/1520-0469(1989)046<2178: NTMITC $>2.0 . \mathrm{CO} ; 2$.
Ertel, H., 1942: Der Vertikale Turbulenz-Wärmestorm in der Atmosphäre. Meteor. Z., 59, 250-253.

Fedorovich, E., and R. Kaiser, 1998: Wind tunnel model study of turbulence regime in the atmospheric convective boundary layer. Buoyant Convection in Geophysical Flows, E. J. Plate et al., Eds., NATO ASI Series, Vol. 513, Kluwer Academic Publishers, $491 \mathrm{pp}$.

Frech, M., and L. Mahrt, 1995: A two-scale mixing formulation for the atmospheric boundary layer. Bound.-Layer Meteor., 73, 91-104, https://doi.org/10.1007/BF00708931.

Garcia, J. R., and J. P. Mellado, 2014: The two-layer structure of the entrainment zone in the convective boundary layer. J. Atmos. Sci., 71, 1935-1955, https://doi.org/10.1175/JAS-D-13-0148.1.

Ghannam, K., T. Duman, S. T. Salesky, M. Chamecki, and G. Katul, 2017: The non-local character of turbulence asymmetry in the convective atmospheric boundary layer. Quart. J. Roy. Meteor. Soc., 143, 494-507, https://doi.org/10.1002/qj.2937.

Gibbs, J. A., E. Fedorovich, and A. M. J. van Eijk, 2011: Evaluating Weather Research and Forecasting (WRF) model predictions of turbulent flow parameters in a dry convective boundary layer. J. Appl. Meteor. Climatol., 50, 2429-2444, https:// doi.org/10.1175/2011JAMC2661.1.

Green, B. W., and F. Zhang, 2015: Idealized large-eddy simulations of a tropical cyclonelike boundary layer. J. Atmos. Sci., 72, 1743-1764, https://doi.org/10.1175/JAS-D-14-0244.1.

Holtslag, A. A. M., and C.-H. Moeng, 1991: Eddy diffusivity and countergradient transport in the convective atmospheric boundary layer. J. Atmos. Sci., 48, 1690-1698, https://doi.org/ 10.1175/1520-0469(1991)048<1690:EDACTI >2.0.CO;2.

Hong, S.-Y., Y. Noh, and J. Dudhia, 2006: A new vertical diffusion package with an explicit treatment of entrainment processes. Mon. Wea. Rev., 134, 2318-2341, https://doi.org/10.1175/ MWR3199.1.

Hunt, J. C. R., J. C. Kaimal, and J. E. Gaynor, 1988: Eddy structure in the convective boundary-layer-New measurements and new concepts. Quart. J. Roy. Meteor. Soc., 114, 827-858, https://doi.org/10.1002/qj.49711448202.

Kaiser, R., and E. Fedorovich, 1998: Turbulence spectra and dissipation rates in a wind tunnel model of the atmospheric convective boundary layer. J. Atmos. Sci., 55, 580-594, https://doi.org/ 10.1175/1520-0469(1998)055<0580:TSADRI >2.0.CO;2.

Klemp, J. B., and R. B. Wilhelmson, 1978: The simulation of threedimensional convective storm dynamics. J. Atmos. Sci., 35, 1070-1096, https://doi.org/10.1175/1520-0469(1978)035<1070: TSOTDC $>2.0 . \mathrm{CO} ; 2$.

Lenschow, D. H., and P. L. Stephens, 1980: The role of thermals in the convective boundary layer. Bound.-Layer Meteor., 19, 509-532, https://doi.org/10.1007/BF00122351.

_ J. C. Wyngaard, and W. T. Pennell, 1980: Mean-field and second-moment budgets in a baroclinic, convective boundary layer. J. Atmos. Sci., 37, 1313-1326, https://doi.org/10.1175/ 1520-0469(1980)037<1313:MFASMB >2.0.CO;2.

Moeng, C.-H., 1984: A large-eddy-simulation model for the study of planetary boundary-layer turbulence. J. Atmos. Sci., 41, 2052-2062, https://doi.org/10.1175/1520-0469(1984)041<2052: ALESMF $>2.0 . \mathrm{CO} ; 2$

Noh, Y., W. G. Cheon, S. Y. Hong, and S. Raasch, 2003: Improvement of the k-profile model for the planetary boundary layer based on large eddy simulation data. Bound.-Layer Meteor., 107, 401-427, https://doi.org/10.1023/A:1022146015946.

Park, S.-B., P. Gentine, K. Schneider, and M. Farge, 2016: Coherent structures in the boundary and cloud layers: Role of updrafts, subsiding shells, and environmental subsidence. 
J. Atmos. Sci., 73, 1789-1814, https://doi.org/10.1175/JAS-D15-0240.1.

Pergaud, J., V. Masson, S. Malardel, and F. Couvreux, 2009: A parameterization of dry thermals and shallow cumuli for mesoscale numerical weather prediction. Bound.-Layer Meteor., 132, 83-106, https://doi.org/10.1007/s10546-009-9388-0.

Plant, R. S., and J.-I. Yano, 2015: Theoretical Background and Formulation. Vol. 1, Parameterization of Atmospheric Convection, Imperial College Press, $1150 \mathrm{pp}$.

Priestley, C. H. B., and W. C. Swinbank, 1947: Vertical transport of heat by turbulence in the atmosphere. Proc. Roy. Soc. London, 189A, 543-561, https://doi.org/10.1098/rspa.1947.0057.

Romps, D. M., and A. B. Charn, 2015: Sticky thermals: Evidence for a dominant balance between buoyancy and drag in cloud updrafts. J. Atmos. Sci., 72, 2890-2901, https://doi.org/10.1175/ JAS-D-15-0042.1.

Salesky, S. T., M. Chamecki, and E. Bou-Zeid, 2017: On the nature of the transition between roll and cellular organization in the convective boundary layer. Bound.-Layer Meteor., 163, 41-68, https://doi.org/10.1007/s10546-016-0220-3.

Schmidt, H., and U. Schumann, 1989: Coherent structure of the convective boundary-layer derived from large-eddy simulations. J. Fluid Mech., 200, 511-562, https://doi.org/10.1017/ S0022112089000753.

Shin, H. H., and S.-Y. Hong, 2013: Analysis of resolved and parameterized vertical transports in convective boundary layers at gray-zone resolutions. J. Atmos. Sci., 70, 3248-3261, https:// doi.org/10.1175/JAS-D-12-0290.1.

— and - 2015: Representation of the subgrid-scale turbulent transport in convective boundary layers at gray-zone resolutions. Mon. Wea. Rev., 143, 250-271, https://doi.org/10.1175/ MWR-D-14-00116.1.

Siebesma, A. P., P. M. M. Soares, and J. Teixeira, 2007: A combined eddy-diffusivity mass-flux approach for the convective boundary layer. J. Atmos. Sci., 64, 1230-1248, https://doi.org/ 10.1175/JAS3888.1.

Sorbjan, Z., 2009: Improving non-local parameterization of the convective boundary layer. Bound.-Layer Meteor., 130, 57-69, https://doi.org/10.1007/s10546-008-9331-9.

Stevens, B., 2000: Quasi-steady analysis of a PBL model with an eddy-diffusivity profile and nonlocal fluxes. Mon. Wea. Rev., 128, 824-836, https://doi.org/10.1175/1520-0493(2000)128<0824: QSAOAP $>2.0 . \mathrm{CO} ; 2$.

Stull, R. B., 1988: An Introduction to Boundary Layer Meteorology. Kluwer Academic, 666 pp.

1993: Review of non-local mixing in turbulent atmospheres: Transilient turbulence theory. Transport and Diffusion in Turbulent Fields, H. Kaplan et al., Eds., Springer Netherlands, 21-96.
Sullivan, P. P., and E. G. Patton, 2011: The effect of mesh resolution on convective boundary layer statistics and structures generated by large-eddy simulation. J. Atmos. Sci., 68, 23952415, https://doi.org/10.1175/JAS-D-10-05010.1.

Svensson, G., and Coauthors, 2011: Evaluation of the diurnal cycle in the atmospheric boundary layer over land as represented by a variety of single-column models: The second GABLS experiment. Bound.-Layer Meteor., 140, 177-206, https:// doi.org/10.1007/s10546-011-9611-7.

Therry, G., and P. Lacarrère, 1983: Improving the eddy kinetic energy model for planetary boundary layer description. Bound.-Layer Meteor., 25, 63-88, https://doi.org/10.1007/ BF00122098.

Troen, I. B., and L. Mahrt, 1986: A simple-model of the atmospheric boundary-layer; sensitivity to surface evaporation. Bound.-Layer Meteor., 37, 129-148, https://doi.org/10.1007/ BF00122760.

Wang, W., X. Shen, and W. Huang, 2016: A comparison of boundary-layer characteristics simulated using different parametrization schemes. Bound-Layer Meteor., 161, 375-403, https://doi.org/10.1007/s10546-016-0175-4.

Warner, J., and J. W. Telford, 1967: Convection below cloud base. J. Atmos. Sci., 24, 374-382, https://doi.org/10.1175/1520-0469 (1967)024<0374:CBCB >2.0.CO;2.

Wyngaard, J. C., 2004: Toward numerical modeling in the "terra incognita." J. Atmos. Sci., 61, 1816-1826, https://doi.org/ 10.1175/1520-0469(2004)061<1816:TNMITT>2.0.CO;2.

_ 2010: Turbulence in the Atmosphere. Cambridge University Press, 393 pp.

Xue, M., K. K. Droegemeier, and V. Wong, 2000: The Advanced Regional Prediction System (ARPS)-A multi-scale nonhydrostatic atmospheric simulation and prediction model. Part I: Model dynamics and verification. Meteor. Atmos. Phys., 75, 161-193, https://doi.org/10.1007/s007030070003.

_ , and Coauthors, 2001: The Advanced Regional Prediction System (ARPS)-A multi-scale nonhydrostatic atmospheric simulation and prediction tool. Part II: Model physics and applications. Meteor. Atmos. Phys., 76, 143-165, https:// doi.org/10.1007/s007030170027.

Young, G. S., 1988: Turbulence structure of the convective boundary layer. Part II: Phoenix 78 aircraft observations of thermals and their environment. J. Atmos. Sci., 45, 727-735, https:// doi.org/10.1175/1520-0469(1988)045<0727:TSOTCB >2.0.CO;2.

Zilitinkevich, S., V. M. Gryanik, V. N. Lykossov, and D. V. Mironov, 1999: Third-order transport and nonlocal turbulence closures for convective boundary layers. J. Atmos. Sci., 56, 3463-3477, https://doi.org/10.1175/1520-0469(1999)056<3463: TOTANT $>2.0 . \mathrm{CO} ; 2$. 